Chapter 10

\title{
Epidural Lysis of Adhesions and Percutaneous Neuroplasty
}

\author{
Gabor B. Racz, James E. Heavner, Jeffrey P. Smith, Carl E. Noe, \\ Adnan Al-Kaisy, Tomikichi Matsumoto, Sang Chul Lee and Laszlo Nagy \\ Additional information is available at the end of the chapter
}

http://dx.doi.org/10.5772/58753

\section{Introduction}

Chances are relatively high that each of us will experience low back pain at some point in our lives. The usual course is rapid improvement with $5 \%$ to $10 \%$ developing persistent symptoms [1]. In the 1990s the estimated cost of low back pain to the health industry was in the billions of dollars, and with a larger proportion of our population now reported to be older, this number can only be expected to increase [2,3]. Treatment typically begins with conservative measures such as medication and physical therapy and may even include minimally and highly invasive pain management interventions. Surgery is sometimes required in patients who have progressive neurologic deficits or those who do not respond to conservative treatment sometimes chose surgery. A quandary sometimes arises, following a primary surgery, as to whether repeat surgery should be attempted or another alternative technique should be tried. This is the exact problem that the epidural adhesiolysis procedure was designed to address. Failed back surgery or postlaminectomy syndrome led to the development of the epidural adhesiolysis procedure. It was shown to be effective in many patients with chronic pain after back surgery presumably by freeing up nerves and breaking down scar formation, delivering site-specific corticosteroids and local anesthetics, and reducing edema with the use of hyaluronidase and hypertonic saline. Epidural adhesiolysis has afforded patients a reduction in pain and neurologic symptoms without the expense and occasional long recovery period associated with repeat surgery, and often prevents the need for surgical intervention. Epidural adhesiolysis was given an evidence rating of strong correlating to a $1 \mathrm{~B}$ or $1 \mathrm{C}$ evidence level for post-lumbar surgery syndrome in the most recent American Society of Interventional Pain Physicians evidence-based guidelines. The therapy is supported by observational studies and case series along with randomized-control trials. The recommendation was also made that this therapy could apply to most patients with post laminectomy syndrome or failed back syn- 
drome in many circumstances with informed consent [4]. Additionally, current procedural terminology (CPT) codes have been assigned to the two different kinds of adhesiolysis: CPT 62263 for the three-times injections over 2 to 3 days, which has recently changed to 3 injections 6- 8 hours apart within 24 hours, usually done in an inpatient hospital setting, and CPT 62264 for the one-time injection series surgery-center model that may need to be repeated 3 to 3.5 times in a 12-month period.

\section{Pathophysiology of epidural fibrosis (scar tissue) as a cause of low back pain with radiculopathy}

The etiology of chronic low back pain with radiculopathy after appropriate surgery is not well understood. Kuslich et al [5] addressed this issue when they studied 193 patients who had undergone lumbar spine operations given local anesthesic into the epidural space. It was postulated that sciatica could only be produced by stimulation of a swollen, stretched, restricted (i.e., scarred) or compressed nerve root [5]. Back pain could be produced by stimulation of several tissues, but the most common tissue of origin was the outer layer of the annulus fibrosus and the posterior longitudinal ligament. Stimulation for pain generation of the facet joint capsule rarely generated low back pain, and facet synovium and cartilage surfaces of the facet or muscles were never tender [6].

The contribution of fibrosis to the etiology of low back pain has been debated [7-9]. There are many possible etiologies of epidural fibrosis, including surgical trauma, an annular tear, infection, hematoma, or intrathecal contrast material [10]. These etiologies have been well documented in the literature. LaRocca and Macnab [11] demonstrated the invasion of fibrous connective tissue into postoperative hematoma as a cause of epidural fibrosis, and Cooper et al [12] reported periradicular fibrosis and vascular abnormalities occurring with herniated intervertebral disks. McCarron et al [13] investigated the irritative effect of nucleus pulposus on the dural sac, adjacent nerve roots, and nerve root sleeves independent of the influence of direct compression on these structures. Evidence of an inflammatory reaction was identified by gross inspection and microscopic analysis of spinal cord sections after homogenized autogenous nucleus pulposus was injected into the lumbar epidural space of four dogs. In the control group consisting of four dogs injected with normal saline, the spinal cord sections were grossly normal. Parke and Watanabe [14] showed significant evidence of adhesions in cadavers with lumbar disk herniation.

It is widely accepted that postoperative scar renders the nerve susceptible to injury by a compressive phenomena [9]. It is natural for connective tissue or any kind of scar tissue to form fibrous layers (scar tissue) as a part of the process that transpires after disruption of the intact milieu [15]. Scar tissue is generally found in three components of the epidural space. Dorsal epidural scar tissue is formed by reabsorption of surgical hematoma and may be involved in pain generation [16]. In the ventral epidural space, dense scar tissue is formed by ventral defects in the disk, which may persist despite surgical treatment and continue to produce low back pain and radiculopathy past the surgical healing phase [17]. The lateral epidural space includes the epiradicular structures outside the root canals, known as the lateral 
recesses or "sleeves," which are susceptible to lateral disk defects, facet hypertrophy, and neuroforaminal stenosis [18].

Although scar tissue itself is not tender, an entrapped nerve root is. Kuslich et al [5] surmised that the presence of scar tissue compounded the pain associated with the nerve root by fixing it in one position and thus increasing the susceptibility of the nerve root to tension or compression. They also concluded that no other tissues in the spine are capable of producing leg pain. In a study of the relationship between peridural scar evaluated by magnetic resonance imaging (MRI) and radicular pain after lumbar diskectomy, Ross et al [19] demonstrated that subjects with extensive peridural scarring were 3.2 times more likely to experience recurrent radicular pain.

This evidence also parallels a new study by Gilbert et al [20] in which lumbosacral nerve roots were identified as undergoing less strain than previously published during straight leg raise and in which hip motion greater than 60 degrees was determined to cause displacement of the nerve root in the lateral recess.

\section{Fluid foraminotomy: Foraminal adhesiolysis or disentrapment}

Relative or functional foraminal root entrapment syndrome secondary to epidural fibrosis with corresponding nerve root entrapment is frequently evident after an epidurogram and signified by lack of epidural contrast flow into epidural finger projections at those levels. The lysis procedure effectively serves as a fluid foraminotomy reducing foraminal stenosis caused by epidural fibrosis. In addition to increasing foraminal cross-sectional area, adhesiolysis serves to decompress distended epidural venous structures that may exert compression at nearby spinal levels (Figures 1 and 2) and inevitably cause needle stick related epidural hematomas. Adhesiolysis has led to the development of flexible epiduroscopy that is being pioneered by, primarily initiated, pursued and to this day supported by Dr. James Heavner [21,22].

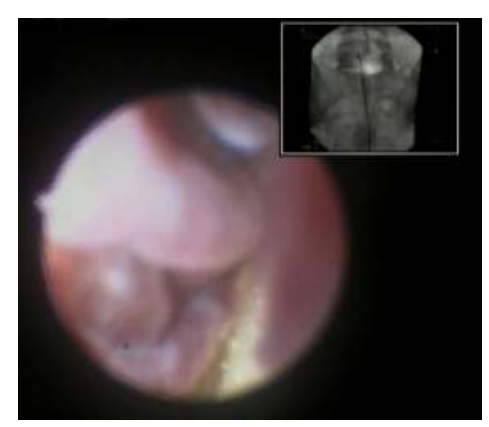

Figure 1. Engorged blood vessels in the epidural cavity as observed during epiduroscopy. Insert in upper right corner is fluoroscopy showing location for epiduroscopy tip (left anterior border of L5). 


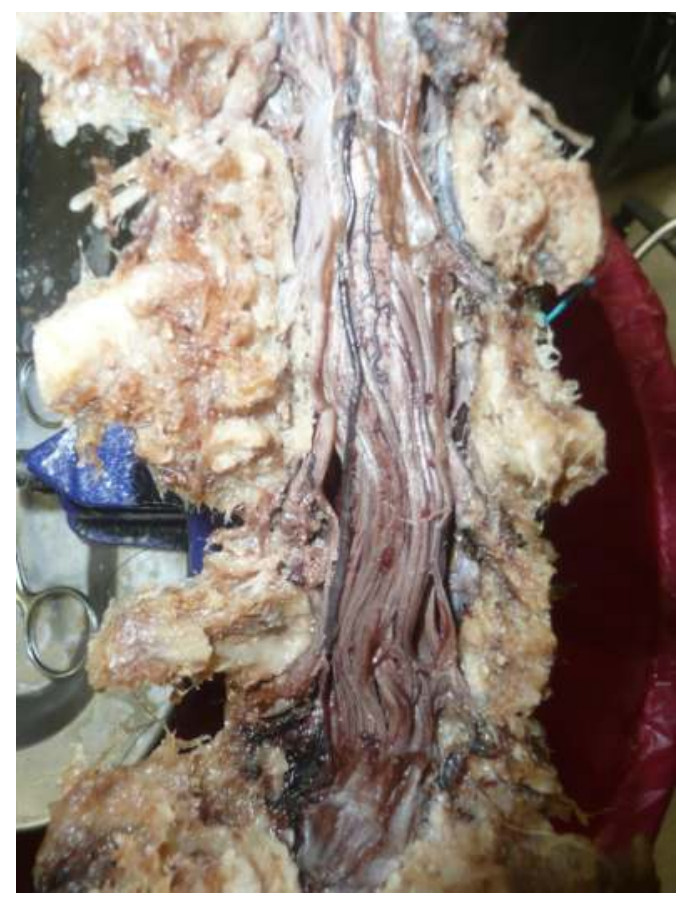

Figure 2. Engorged blood vessels in the epidural cavity in cadaver. See vein on right side next to the nerve root target site for fluid foraminotomy and opening venous run off and decompression.

\section{Diagnosis and radiologic diagnosis of epidural fibrosis}

As with any patient, a thorough musculoskeletal and neurologic examination should be performed. In addition to standard dural tension provocative tests, we recommend a provocative test called 'dural tug.' To perform the test, the patient should be instructed to sit up with a straight leg, bend forward flexing the lumbar spine until their back pain starts to become evident, and the head and neck flexed rapidly forward. During this maneuver, the dura is stretched cephalad and if adhered to structures such as the posterior longitudinal ligament, the most heavily innervated spinal canal structure, the movement of the dura will elicit back pain that is localized to the pain generator. A positive dural tug maneuver has been observed to resolve after percutaneous neuroplasty. (Figures 3-7). 


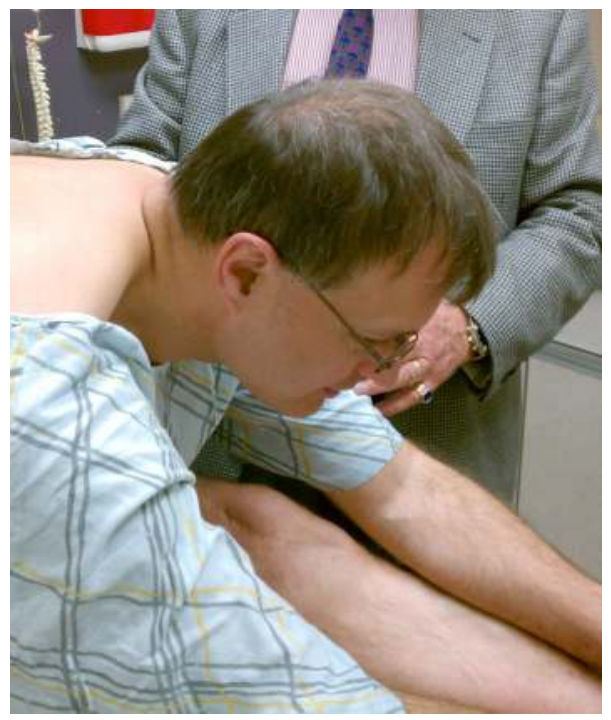

Figure 3. The 'dural tug' maneuver being performed prior to percutaneous neuroplasty.

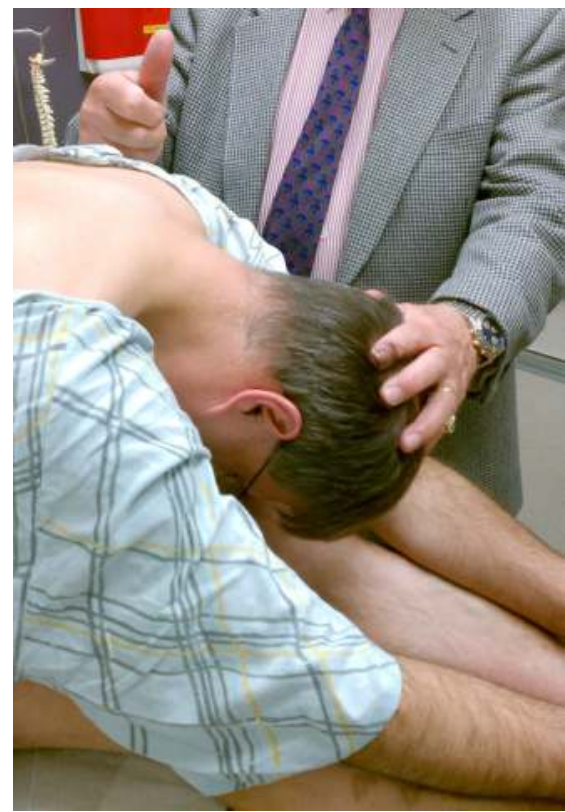

Figure 4. Note pain reproduction prior to full neck flexion secondary to dural adhesions. 


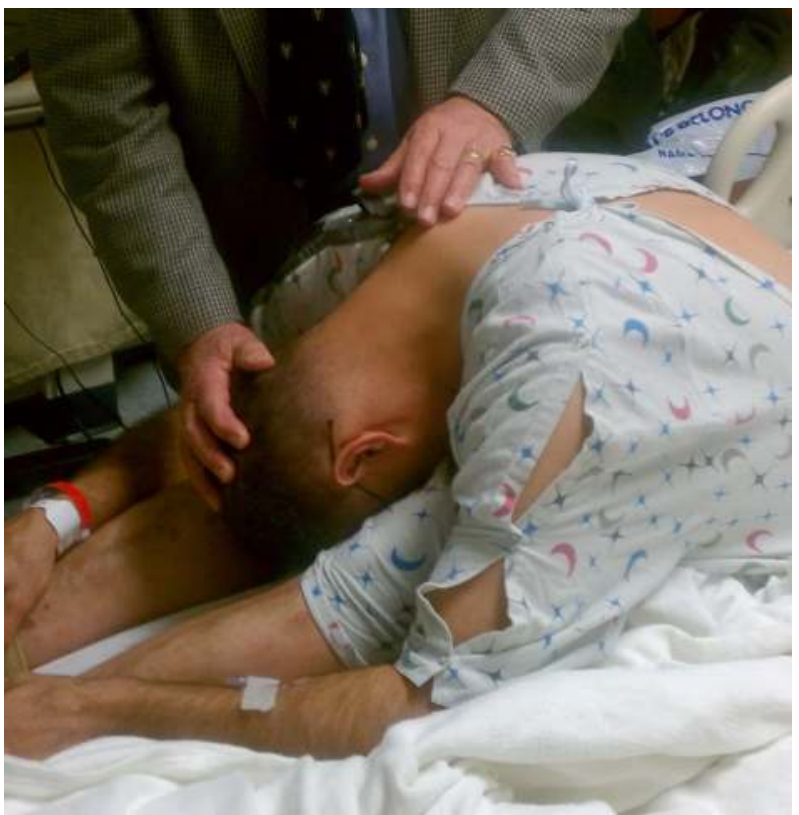

Figure 5. Patient after percutaneous neuroplasty with pain free neck and back flexion due to treatment of dural adhesions.

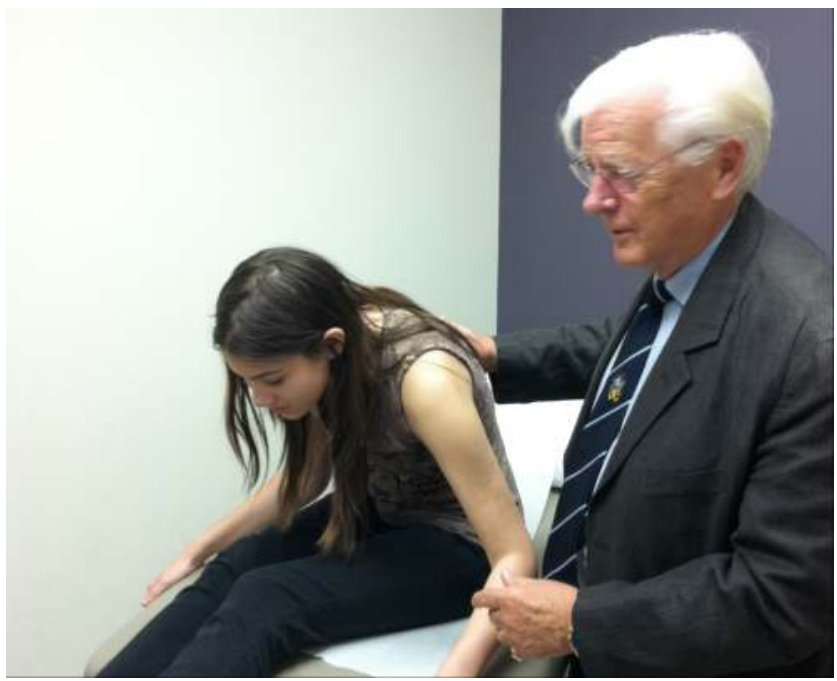

Figure 6. There is decreased spine flexion prior to treatment secondary to dural adhesions. The pain generator was subsequently documented to be T9-T10 dural adhesion from an annular tear. 


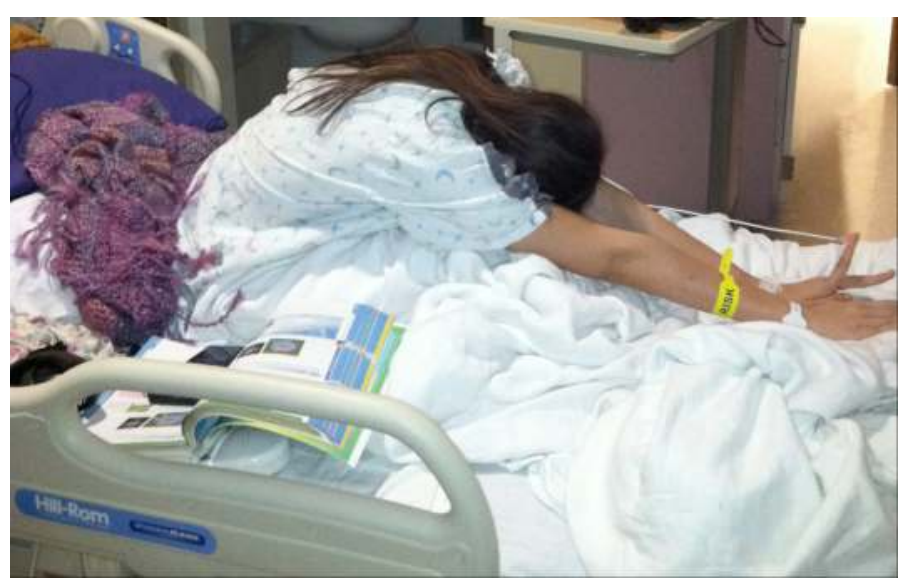

Figure 7. After treatment, the same patient demonstrates increased painless flexion of the spine.

MRI and computed tomography (CT) are diagnostic tools; sensitivity and specificity are $50 \%$ and $70 \%$, respectively [15]. CT myelography may also be helpful, although none of the aforementioned modalities can identify epidural fibrosis with $100 \%$ reliability. In contrast, epidurography is a technique used with considerable success and it is believed that epidural fibrosis is best diagnosed by performing an epidurogram [23-26]. It can detect filling defects in good correlation with a patient's symptoms in real time [26]. A combination of several of these techniques would undoubtedly increase the ability to identify epidural fibrosis.

\section{1. current procedural terminology or CPT codes}

The American Medical Association has developed Current Procedural Terminology codes for epidural adhesiolysis, which include 62264 for a single infusion and 62263 for a staged threeseries infusion.

\subsection{Indications for epidural adhesiolysis}

Although originally designed to treat radiculopathy secondary to epidural fibrosis following surgery, the use of epidural adhesiolysis has been expanded to treat a multitude of pain etiologies. These include the following [27]:

1. Failed back surgery syndrome

2. Postlaminectomy syndrome of the neck and back after surgery

3. Disk disruption

4. Metastatic carcinoma of the spine leading to compression fracture

5. Multilevel degenerative arthritis

6. Facet pain 
7. Spinal stenosis

8. Pain unresponsive to spinal cord stimulation and spinal opioids

9. Thoracic disk related chest wall and abdominal pain (after mapping)

\subsection{Contraindications}

The following are absolute contraindications for performing epidural adhesiolysis:

1. Sepsis

2. Chronic infection

3. Coagulopathy

4. Local infection at the procedure site

5. Patient refusal

6. Syrinx formation

A relative contraindication is the presence of arachnoiditis. With arachnoiditis, the tissue planes may be adherent to one another, increasing the chance of loculation of contrast or medication. It may also increase the chance of spread of the medications to the subdural or subarachnoid space, which can increase the chance of complications. Practitioners with limited experience with epidural adhesiolysis should consider referring these patients to a clinician with more training and experience.

\section{Patient preparation}

When epidural adhesiolysis has been deemed an appropriate treatment modality, the risks and benefits of the procedure should be discussed with the patient and informed consent obtained. The benefits are pain relief, improved physical function, and possible reversal of neurologic symptoms. Risks include, but are not limited to, bruising, bleeding, infection, reaction to medications used (i.e., hyaluronidase, local anesthetic, corticosteroids, hypertonic saline), damage to nerves or blood vessels, no or little pain relief, bowel/bladder incontinence, worsening of pain, and paralysis. Patients with a history of urinary incontinence should have an urodynamic evaluation by a urologist before the procedure to document the preexisting urodynamic etiology and pathology.

\section{Anticoagulant medication}

Medications that prolong bleeding and clotting parameters should be withheld before performing epidural adhesiolysis. The length of time varies depending on the medication taken. A consultation with the patient's primary physician should be obtained before stopping 
any of these medications, particularly in patients who require chronic anticoagulation such as those with drug-eluting heart stents or prosthetic heart valves. Nonsteroidal anti-inflammatory drugs and aspirin, respectively, should be withheld 4 days and 7 to 10 days before the procedure. Although there is much debate regarding these medications and neuraxial procedures, we tend to be on the conservative side. Clopidogrel (Plavix) should be stopped 7 days before, whereas ticlopidine (Ticlid) is withheld 10 to 14 days before the adhesiolysis [28]. Warfarin (Coumadin) stoppage is variable but 5 days is usually adequate [27]. Patients on subcutaneous heparin should have it withheld a minimum of 12 hours before the procedure, whereas those on low-molecular-weight heparin require a minimum of 24 hours [28]. Overthe-counter homeopathic medications that prolong bleeding parameters should also be withheld. These include fish oil, vitamin E, gingko biloba, garlic, ginseng, and St. John's Wort. Adequate coagulation status can be confirmed by the history, INR, prothrombin time, partial thromboplastin time, and a platelet function assay or bleeding time. The tests should be performed as close to the day of the procedure as possible. Tests performed only a few days after stopping the anticoagulant medication may come back elevated because not enough time has elapsed to allow the anticoagulant effects of the medication to resolve. The benefits of the procedure must be weighed against the potential sequelae of stopping the anticoagulant medication and this should be discussed thoroughly with the patient.

\section{Preoperative laboratory}

Before the procedure, a complete blood count and a clean-catch urinalysis are obtained to check for any undiagnosed infections. An elevated white count and/or a positive urinalysis should prompt the physician to postpone the procedure and refer the patient to the primary care physician for further workup and treatment. In addition, history of bleeding, abnormalities a prothrombin time, partial thromboplastin time, and platelet function assay or bleeding time, are obtained to check for coagulation abnormalities. Any elevated value warrants further investigation and postponement of the procedure until those studies are complete.

\section{Technique}

This procedure can be performed in the cervical, thoracic, lumbar, and caudal regions of the spine. The caudal and transforaminal placement of catheters will be described in detail, whereas highlights and slight changes in protocol will be provided for cervical and thoracic catheters. Our policy is to perform this procedure under strict sterile conditions in the operating room. Prophylactic antibiotics with broad neuraxial coverage are given before the procedure. Patients will receive either ceftriaxone $1 \mathrm{~g}$ intravenously or Levaquin $500 \mathrm{mg}$ orally in those allergic to penicillin. The same dose is also given on day 2. An anesthesiologist or nurse anesthetist provides monitored anesthesia care. 


\section{Caudal approach}

The patient is placed in the prone position with a pillow placed under the abdomen to correct the lumbar lordosis and a pillow under the ankles for patient comfort. The patient is asked to put his or her toes together and heels apart. This relaxes the gluteal muscles and facilitates identification of the sacral hiatus. After sterile preparation and draping, the sacral hiatus is identified via palpation just caudal to the sacral cornu or with fluoroscopic guidance. A skin wheal is raised with local anesthetic 1-inch lateral and 2 inches caudal to the sacral hiatus on the side opposite the documented radiculopathy. A distal subcutaneous approach theoretically provides some protection from meningitis, as a local skin infection would be much preferred over infection closer to the caudal epidural space. The skin is nicked with an 18-gauge cutting needle, and a 15-or 16-gauge RX Coudé (Epimed International) epidural needle is inserted through the nick at a 45-degree angle and guided fluoroscopically or by palpation to the sacral hiatus (Figures 8 and 9).

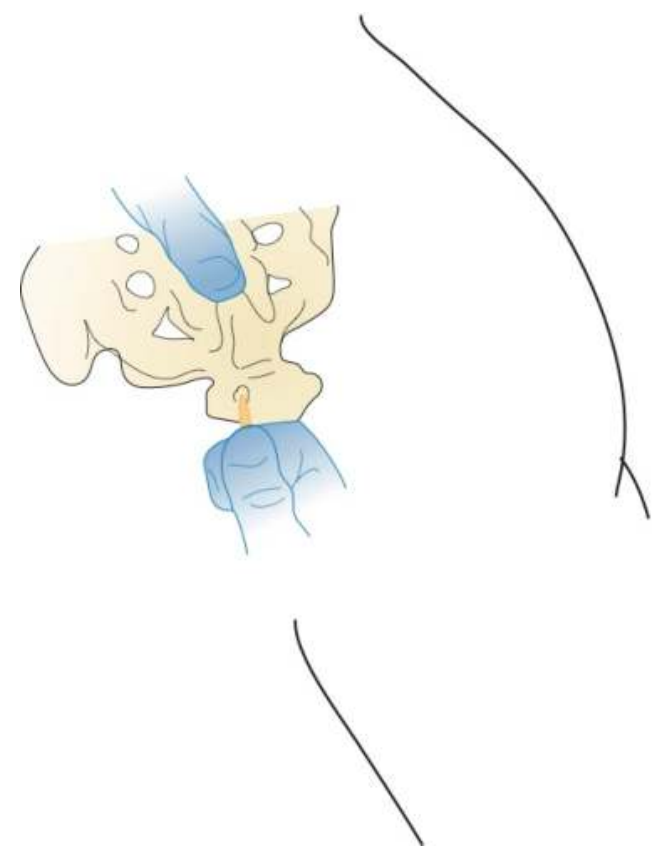

Figure 8. Caudal lysis sequence-first find sacral hiatus and tip of coccyx.

When the needle is through the hiatus, the angle of the needle is dropped to approximately 30 degrees and advanced. The advantages of the RX Coudé needle over other needles are the angled tip, which enables easier direction of the catheter, and the tip of the needle is less sharp. The back edge of the distal opening of the needle is designed to be a noncutting surface that allows manipulation of the catheter in and out of the needle. A Touhy needle has the back edge 
of the distal opening, which is a cutting surface and can more easily shear a catheter. A properly placed needle will be inside the caudal canal below the level of the S3 foramen on anteroposterior (AP) and later fluoroscopic images. A needle placed above the level of the $\mathrm{S} 3$ foramen could potentially puncture a low-lying dura. The needle tip should cross the midline of the sacrum toward the side of the radiculopathy.

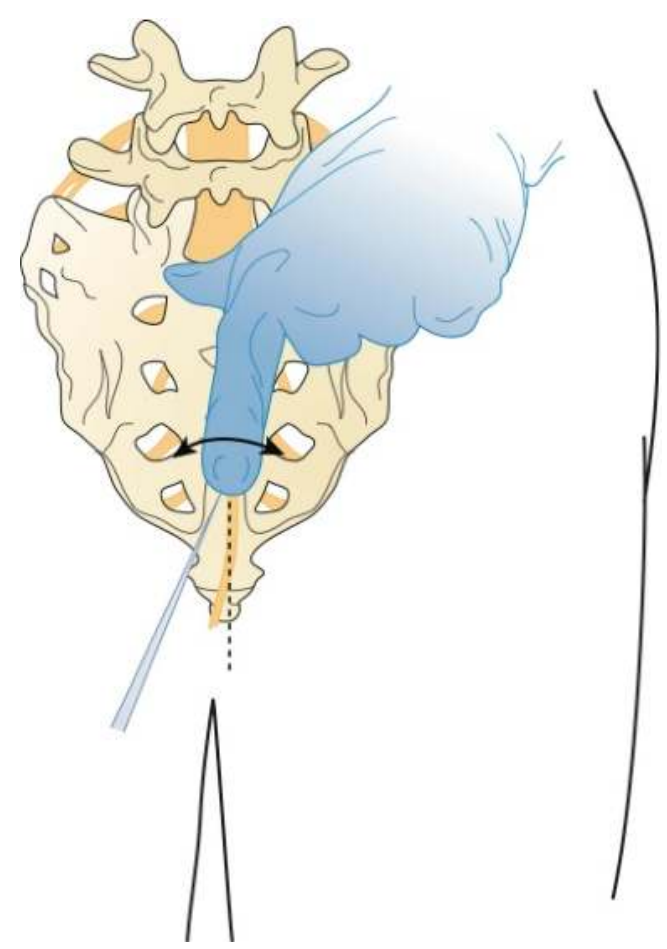

Figure 9. Roll palpating index finger to identify the sacral cornu and thus the target sacral hiatus.

An epidurogram is performed using $10 \mathrm{~mL}$ of a non-ionic, water-soluble contrast agent. Confirm a negative aspiration for blood or cerebrospinal fluid before any injection of the contrast or medication. Omnipaque and Isovue are the two agents most frequently used and are suitable for myelography [29, 30]. Do not use ionic, water-insoluble agents such as Hypopaque or Renografin or ionic, water-soluble agents such as Conray [31,32]. These agents are not indicated for myelography. Accidental subarachnoid injections can lead to serious untoward events such as seizure and possibly death. Slowly inject the contrast agent and observe for filling defects. A normal epidurogram will have a "Christmas tree" pattern with the central canal being the trunk and the outline of the nerve roots making up the branches. An abnormal epidurogram will have areas where the contrast does not fill (Figure 10). These are the areas of presumed scarring and typically correspond to the patient's radicular complaints. If vascular uptake is observed, the needle needs to be redirected. 


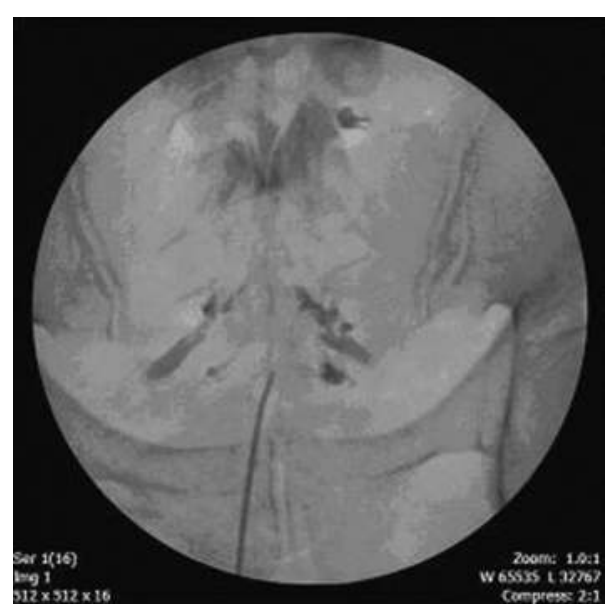

Figure 10. Initial dye injection Omnipaque $240(10 \mathrm{~mL})$ showing sacral S3 runoff and filling defects at S2, S1, and right L5.

After turning the distal opening of the needle ventral lateral, insert a TunL Kath or TunL-XL (stiffer) catheter (Epimed International) with a bend on the distal tip through the needle (Figures 11 and 12). The bend should be $2.5 \mathrm{~cm}$ from the tip of the catheter and at a 30-degree angle. The bend will enable the catheter to be steered to the target level (Figure 13). Under continuous AP fluoroscopic guidance, advance the tip of the catheter toward the ventral-lateral epidural space of the desired level. The catheter can be steered by gently twisting the catheter in a clockwise or counterclockwise direction. Avoid "propellering" the tip (i.e., twisting the tip in circles) because this makes it more difficult to direct the catheter. Do not advance the catheter up the middle of the sacrum because this makes guiding the catheter to the ventrallateral epidural space more difficult. Ideal location of the tip of the catheter in the AP projection is in the foramen just below the midportion of the pedicle shadow (Figures 14 and 15). Check a lateral projection to confirm that the catheter tip is in the ventral epidural space.

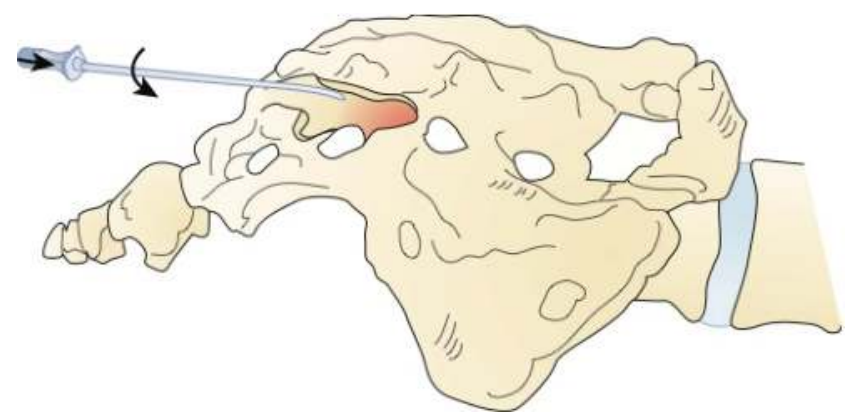

Figure 11. The needle is placed through the sacral hiatus into the sacral canal and rotated in the direction of the target. Do not advance beyond the S3 foramen. 


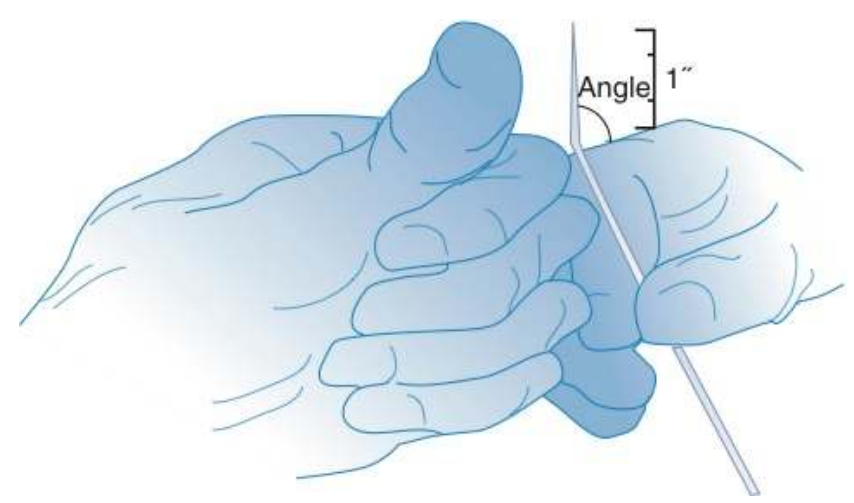

Figure 12. The Epimed Racz catheter is marked for the location of the bend, or use the thumb as reference for the 15degree angle bend.

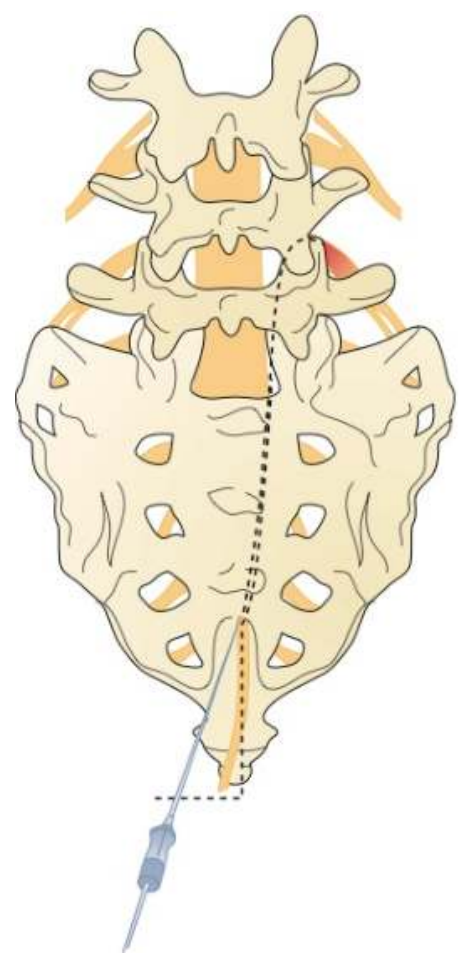

Figure 13. The direction of the catheter is just near the midline; direct the curve under continuous fluoroscopic guidance to the ventral lateral target site. The needle rotation, as well as the catheter navigation, may need to be used to reach the target. 

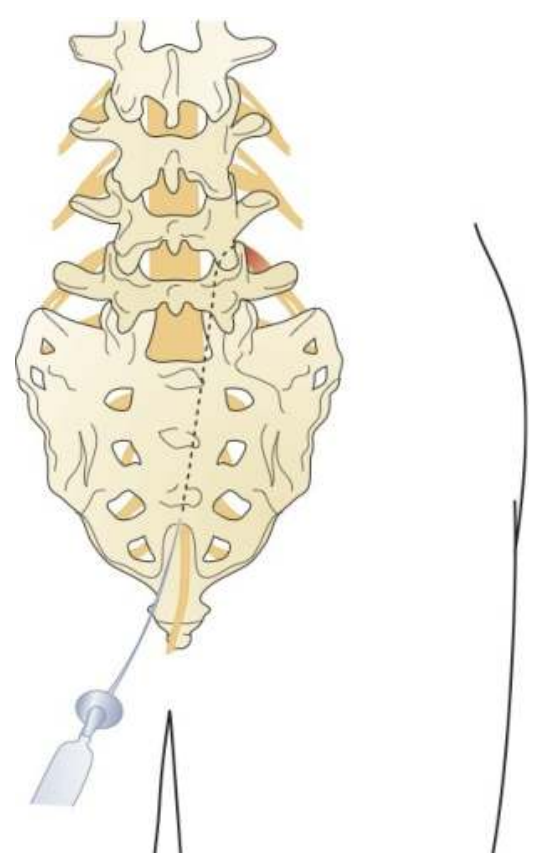

Figure 14. The needle is removed, and the catheter is placed in the ventral lateral epidural space ventral to the nerve root.

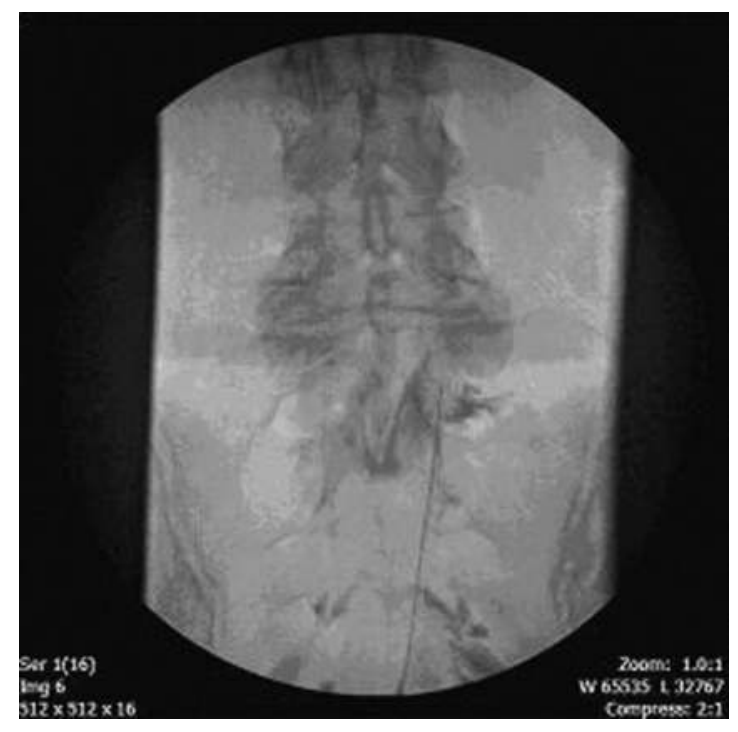

Figure 15. Catheter (24xL) is threaded to lateral L5 neural foramen. 
Under real-time fluoroscopy, inject 2 to $3 \mathrm{~mL}$ of additional contrast through the catheter in an attempt to outline the "scarred in" nerve root (Figure 16). If vascular uptake is noted, reposition the catheter and reinject contrast. Preferably there should not be vascular runoff, but infrequently secondary to venous congestion, an epidural pattern is seen with a small amount of vascular spread. This is acceptable as long as the vascular uptake is venous in nature and not arterial. Extra caution should be taken when injecting the local anesthetic to prevent local anesthetic toxicity. Toxicity is volume and dose related and so far there has not been any reported complications from small volume venous spread. Any arterial spread of contrast always warrants repositioning of the catheter. We have never observed intra-arterial placement in 25 years of placing soft, spring-tipped catheters.

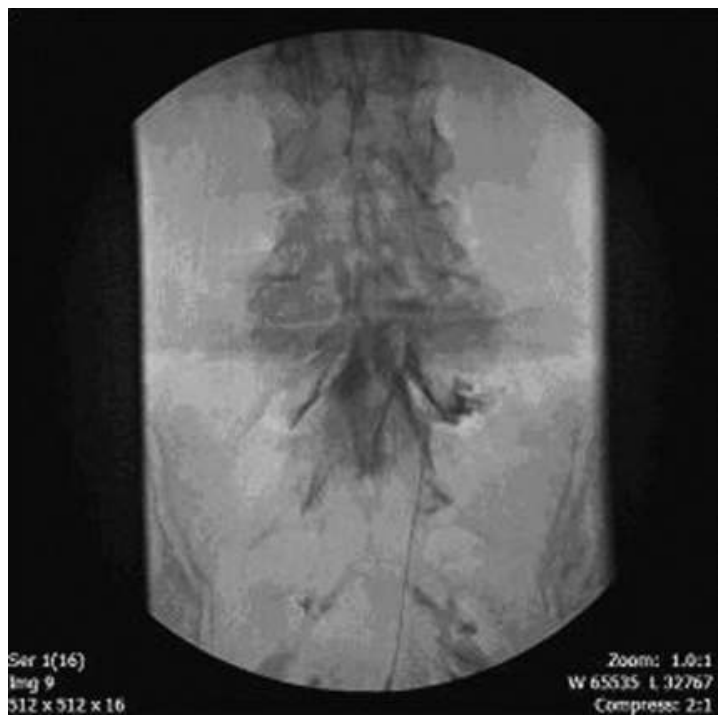

Figure 16. Contrast injection Omnipaque 240, additional $5 \mathrm{~mL}$ opening right L5, S1, S2, and S3 perineural spaces; also left L5, S1, S2, and S3 in addition to right L4 spread in cephalad direction.

Inject $1500 \mathrm{U}$ of hyaluronidase dissolved in $10 \mathrm{~mL}$ of preservative-free normal saline. A newer development is the use of Hylenex or human-recombinant hyaluronidase, which carries the advantage of a reportedly increased effectiveness at the body's normal $\mathrm{pH}$ compared to bovinerecombinant hyaluronidase [33]. This injection may cause some discomfort, so a slow injection is preferable. Observe for "opening up"(i.e. visualization) of the "scarred in" nerve root (Figures 17 and 18 see also Figure 16). A $3 \mathrm{~mL}$ test dose of a $10 \mathrm{~mL}$ local anesthetic/steroid (LA/ S) solution is then given. Our institution used $4 \mathrm{mg}$ of dexamethasone mixed with $9 \mathrm{~mL}$ of $0.2 \%$ ropivacaine. Ropivacaine is used instead of bupivacaine for two reasons: the former produces a preferential sensory versus a motor block, and it is less cardiotoxic than a racemic bupivacaine. Doses for other corticosteroids commonly used are 40 to $80 \mathrm{mg}$ of methylprednisolone (Depo-Medrol), 25 to $50 \mathrm{mg}$ of triamcinolone diacetate (Aristocort), 40 to $80 \mathrm{mg}$ of triamcino- 
lone acetonide (Kenalog), and 6 to $12 \mathrm{mg}$ of betamethasone (Celestone Solu span). If, after 5 minutes, there is no evidence of intrathecal or intravascular injection of medication, inject the remaining $7 \mathrm{~mL}$ of the LA/S solution.

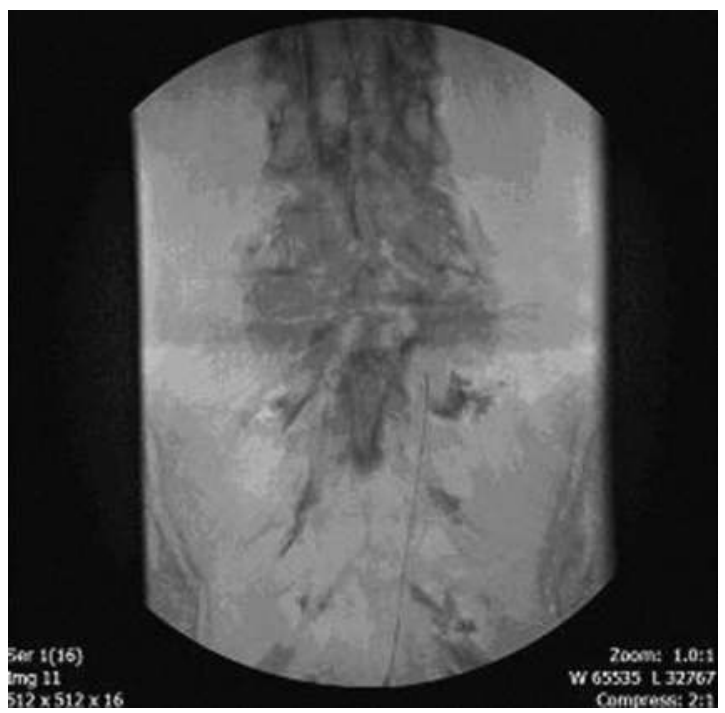

Figure 17. Additional contrast and hyaluronidase injection opens up bilaterally formerly scarred areas. The Christmas tree appearance is obvious.

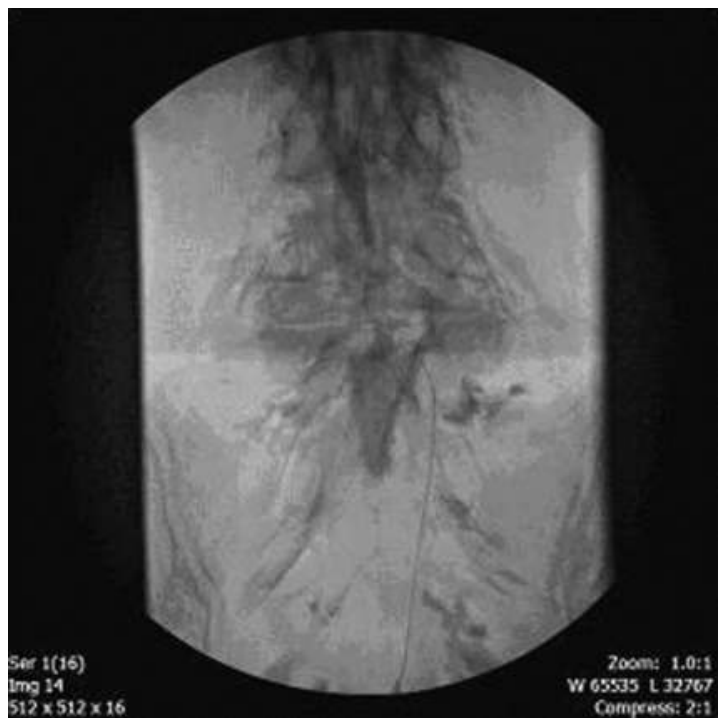

Figure 18. Catheter advances to the desired symptomatic level of right L5 in the ventral lateral epidural space. Injection of contrast followed by $10 \mathrm{~mL}$ hyaluronidase 1,500 units opens up bilaterally L3-5, S1, S2, and S3 neural foramina. 
Remove the needle under continuous fluoroscopic guidance to ensure the catheter remains at the target level (Figure 19). Secure the catheter to the skin using nonabsorbable suture and coat the skin puncture site with antimicrobial ointment. Apply a sterile dressing and attach a 0.2 $\mu \mathrm{m}$ filter to the end of the catheter. Affix the exposed portion of the catheter to the patient with tape and transport the patient to the recovery area.
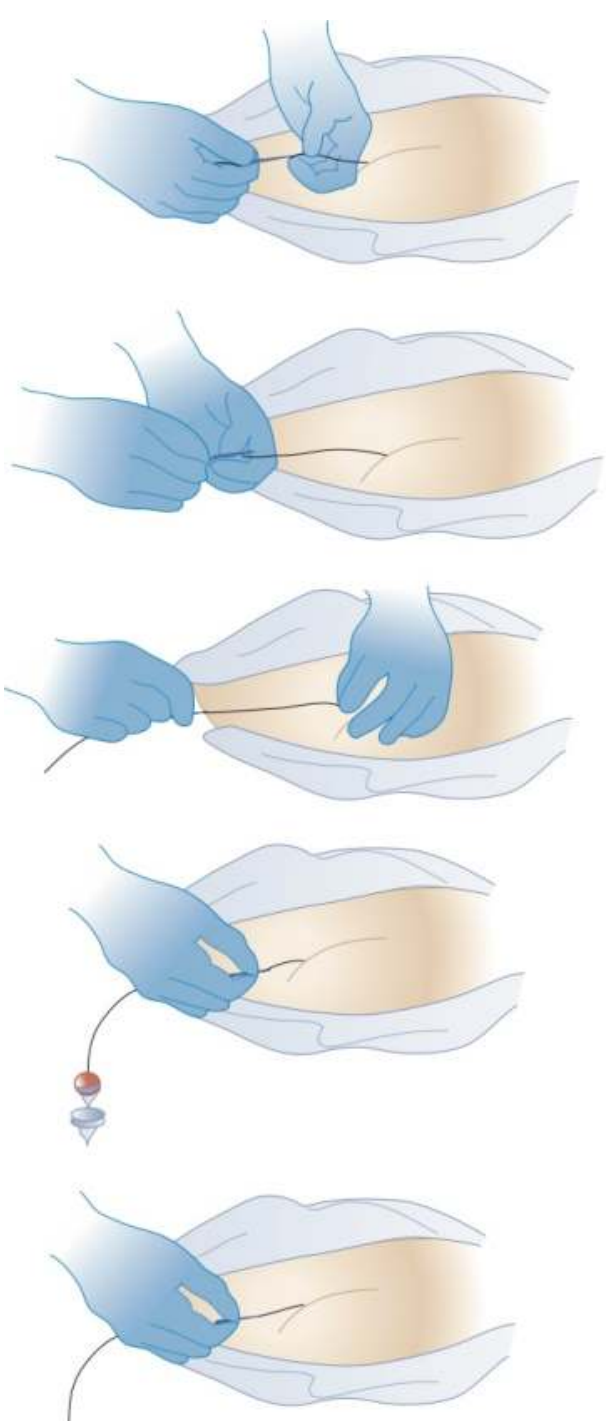

Figure 19. Five picture sequence of removal of the needle to prevent dislodging the catheter from target site before suturing and application of dressing. 
A 20-to 30-minute period should elapse between the last injection of the LA/S solution and the start of the hypertonic saline $(10 \%)$ infusion. This is necessary to ensure that a subdural injection of the LA/S solution has not occurred. A subdural block mimics a subarachnoid block but it takes longer to establish, usually 16 to 18 minutes. Evidence for subdural or subarachnoid spread is the development of motor block. If the patient develops a subarachnoid or subdural block at any point during the procedure, the catheter should be removed and the remainder of the adhesiolysis canceled. The patient needs to be observed to document the resolution of the motor and sensory block and to document that $10 \mathrm{~mL}$ of the hypertonic saline is then infused through the catheter over 15 to 30 minutes. If the patient complains of discomfort, the infusion is stopped and an additional 2 to $3 \mathrm{~mL}$ of $0.2 \%$ ropivacaine is injected and the infusion is restarted. Alternatively, 50 to $75 \mu \mathrm{g}$ of fentanyl can be injected epidurally in lieu of the local anesthetic. After completion of the hypertonic saline infusion, the catheter is slowly flushed with $2 \mathrm{~mL}$ of preservative-free normal saline and the catheter is capped.

Our policy is to admit the patient for 24-hour observation status and do a second and a third hypertonic saline infusion the following day. On post-catheter insertion day 2, the catheter is twice injected (separated by 4-to 6-hour increments) with $10 \mathrm{~mL}$ of $0.2 \%$ ropivacaine without steroid and infused with $10 \mathrm{~mL}$ of hypertonic saline $(10 \%)$ using the same technique and precautions as the day 1 infusion. At the end of the third infusion, the catheter is removed and a sterile dressing applied. The patient is discharged home with 5 days of oral cephalexin at 500 mg twice a day or oral levofloxacin (Levaquin) at $500 \mathrm{mg}$ once a day for penicillin-allergic patients. Clinic follow-up is in 30 days.

\section{Transforaminal catheters}

Patients with an additional level of radiculopathy or those in whom the target level cannot be reached by the caudal approach may require placement of a second catheter. The second catheter is placed into the ventral epidural space via a transforaminal approach.

After the target level is identified with an AP fluoroscopic image, the superior endplate of the vertebra that comprises the caudal portion of the foramina is "squared," that is, the anterior and posterior shadows of the vertebral endplate are superimposed. The angle is typically 15 to 20 degrees in a caudocephalad direction. The fluoroscope is then oblique approximately 15 degrees to the side of the radiculopathy and adjusted until the spinous process is rotated to the opposite side. This fluoroscope positioning allows the best visualization of the superior articular process (SAP) that forms the inferoposterior portion of the targeted foramen. The image of the SAP should be superimposed on the shadow of the disk space on the oblique view. The tip of the SAP is the target for the needle placement (Figure 20). Raise a skin wheal slightly lateral to the shadow of the tip of the SAP. Pierce the skin with an 18-gauge needle and then insert a 15-or 16-gauge RX Coudé needle and advance using gun-barrel technique toward the tip of the SAP. Continue to advance the needle medially toward the SAP until the tip contacts bone. Rotate the tip of the needle 180 degrees laterally and advance about $5 \mathrm{~mm}$ (Figure 21). Rotate the needle back medially 180 degrees (Figure 22). 


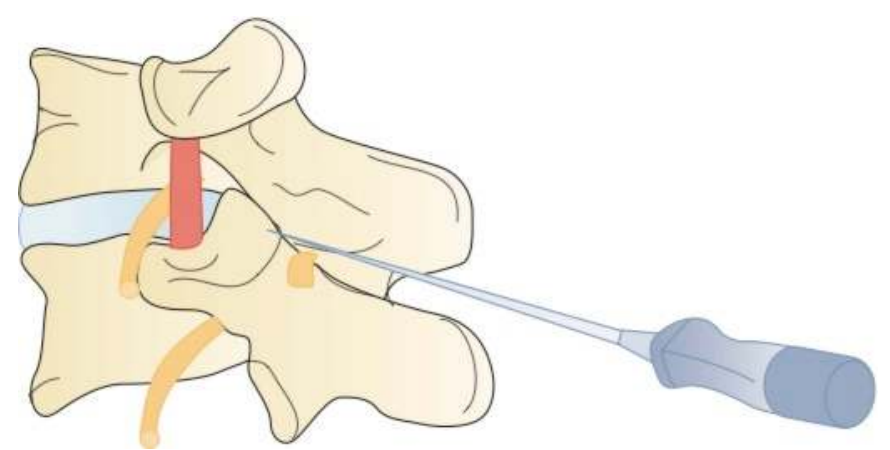

Figure 20. Transforaminal lateral-oblique view. Target the SAP with the advancing RX Coude needle.

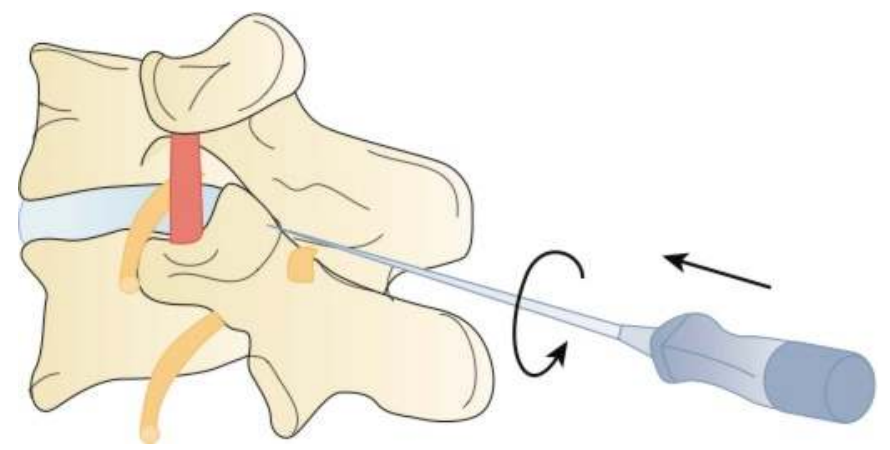

Figure 21. Following bony contact with SAP. Lateral rotation of 180 degrees to allow passage toward the target.

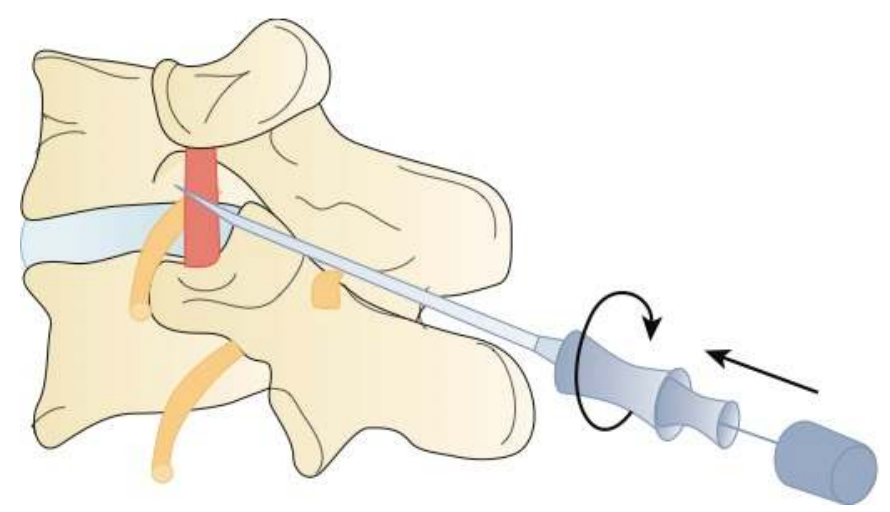

Figure 22. Note the intertransverse ligament. The needle tip with the RX Coude 2 that has 1 mm protruding blunt stylet will pass through the ligament and will be less likely to damage the nerve. 
As the needle is advanced slowly, a clear "pop" is felt as the needle penetrates the intertransverse ligament. Obtain a lateral fluoroscopic image. The tip of the needle should be just past the SAP in the posterior foramen. In the AP plane, the tip of the needle under continuous AP fluoroscopy, insert the catheter slowly into the foramen and advance until the tip should be just short of the middle of the spinal canal (Fig 23-25).

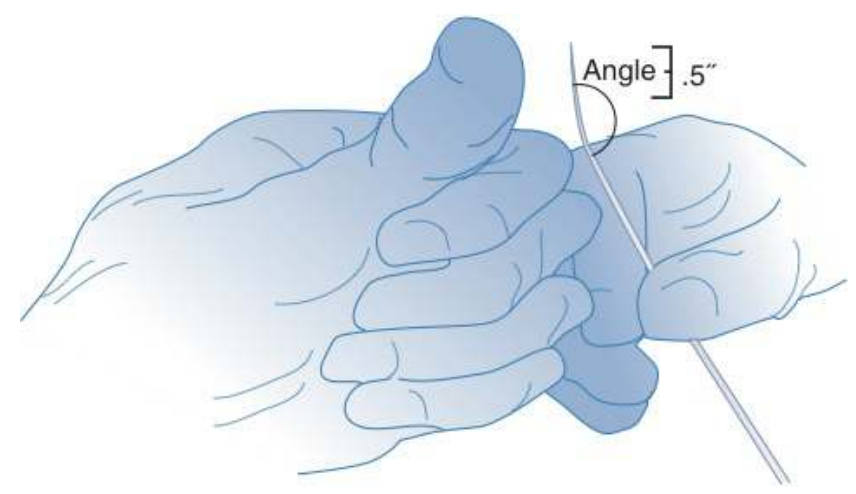

Figure 23. The distal tip of the catheter may be bent 15-degrees, $3 / 4$ inch length.

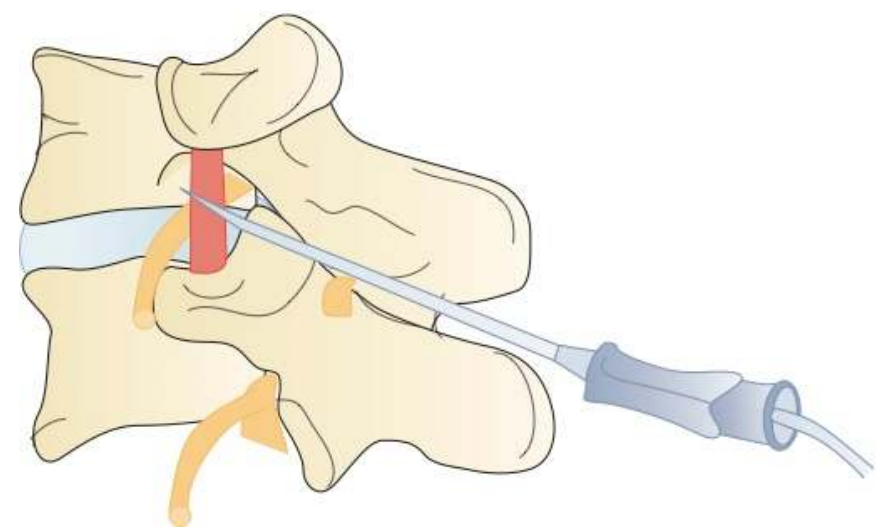

Figure 24. Once the intertransverse ligament is perforated, the catheter is steered to the ventral lateral epidural space (lateral view).

Confirm that the catheter is in the anterior epidural space with a lateral image (Figure 26). Anatomically, the catheter is in the foramen above or below the exiting nerve root (Figure 27). If the catheter cannot be advanced, it usually means the needle is either too posterior or too lateral to the foramen. It can also indicate that the foramen is too stenotic to allow passage of the catheter. The needle can be advanced a few millimeters anteriorly in relation to the foramen, and that will also move it slightly medial into the foramen. If the catheter still will 


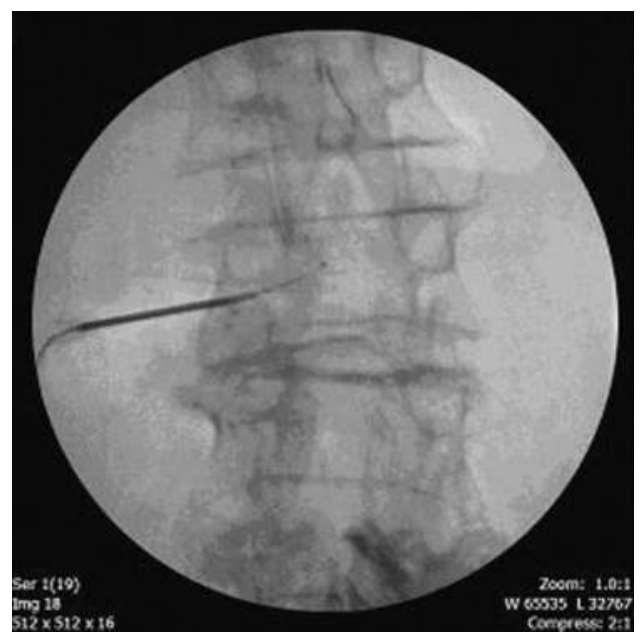

Figure 25. Transforaminal 15-gauge RX-Coude 2 (Epimed International, Johnstown, NY) catheter at left L3-4 threaded almost to near midcanal position (anteroposterior view).

not pass, the initial insertion of the needle will need to be more lateral. Therefore the fluoroscope angle will be about 20 degrees instead of 15 degrees. The curve of the needle usually facilitates easy catheter placement. The final position of the catheter tip is just short of the midline.

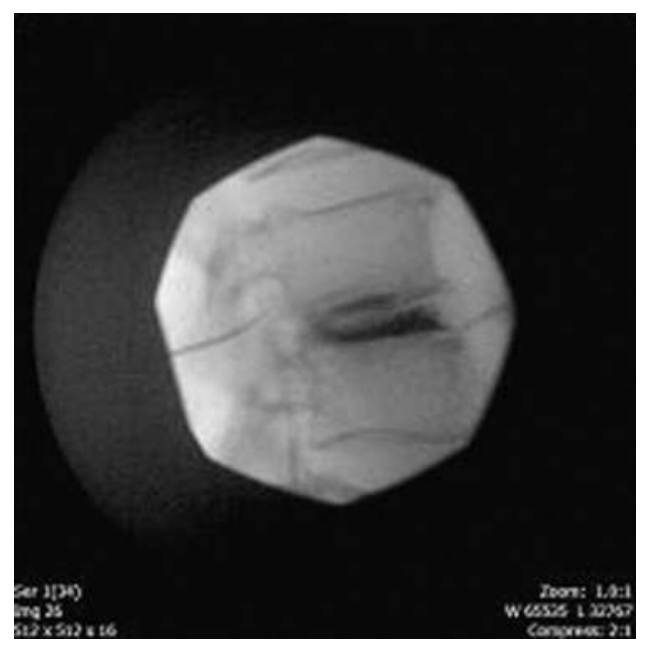

Figure 26. Lateral view of Figure. 16-13. Transforaminal-ventral-anterior catheter dye spread to epidural and L3-4 intradiscal area (through annular tear). 


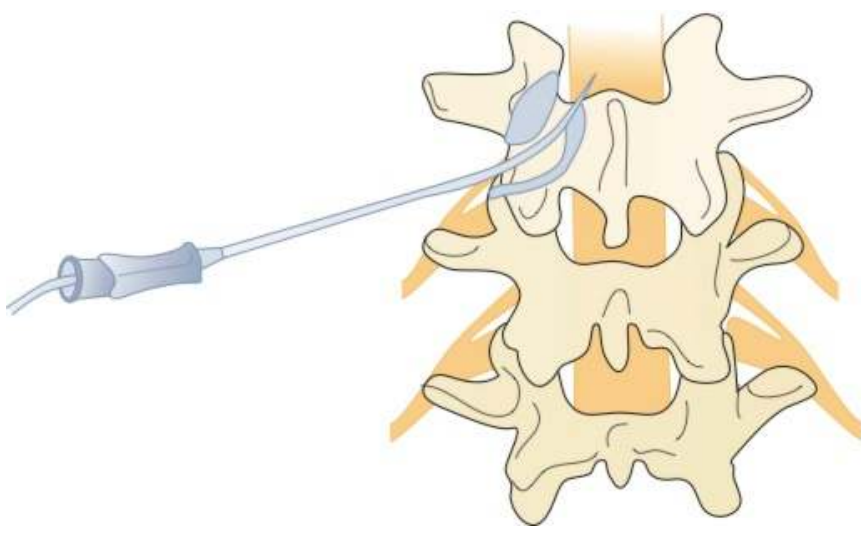

Figure 27. Anteroposterior view. The catheter is in optimal position near midline via the transforaminal placement.

Inject 1 to $2 \mathrm{~mL}$ of contrast to confirm epidural spread. When a caudal and a transforaminal catheter are placed, the $1500 \mathrm{U}$ of hyaluronidase are divided evenly between the two catheters ( $5 \mathrm{~mL}$ of the hyaluronidase/saline solution into each). The LA/S solution is also divided evenly, but a volume of $15 \mathrm{~mL}$ ( $1 \mathrm{~mL}$ steroid and $14 \mathrm{~mL} 0.2 \%$ ropivacaine; of the total volume, $5 \mathrm{~mL}$ is transforaminal and $10 \mathrm{~mL}$ is caudal) is used instead of $10 \mathrm{~mL}$. Remove the needle under fluoroscopic guidance to make sure the catheter does not move from the original position in the epidural space. Secure and cover the catheter as described previously. The hypertonic saline solution is infused at a volume of 4 to $5 \mathrm{~mL}$ per transforaminal and 8 to $10 \mathrm{~mL}$ per caudal catheter over 30 minutes. The hypertonic saline injection volume should always be less than or equal to the local anesthetic volume injected to avoid pain from injection. It behooves the practitioner to check the position of the transforaminal catheter under fluoroscopy before performing the second and third infusions. The catheter may advance across the epidural space into the contralateral foramen or paraspinous muscles or more commonly back out of the epidural space into the ipsilateral paraspinous muscles.

This results in deposition of the medication in the paravertebral tissue rather than in the epidural space. As with the caudal approach, remove the transforaminal catheter after the third infusion. A recent development is the R-X Coude 2 needle in which a second protruding stylet may allow closer needle placement and less chance of nerve injury.

\section{1. $1^{\text {st }}$ sacral foramen approach}

The area at the L5S1 anterolateral epidural space is frequently occupied with epidural adhesions which are associated with pain and a lack of contrast filling on epidurography. This volume of this space has been measured to be $1.1 \mathrm{ml}$ anatomically and $0.9 \mathrm{ml}$ surgically [34]. Lysis of adhesions via the caudal approach may be difficult in patients with epidural adhesions at this location and the S1 foraminal approach may be used to achieve lysis and fluid foraminotomy at this level [35]. 
Matsumoto reported 36 cases with adhesive S-1 radiculopathy. After the procedure, the patients were followed up for 12 months. A marked decrease in VAS and improvement in ADL (improvement in ODI scores) were observed [36].

http://www.paincast.com has video information regarding this procedure [37].

\section{Cervical lysis of adhesions}

The success of the caudal approach for lysis of adhesions led to the application of the same technique to the cervical epidural space. The indications and preprocedure workup are the same as those for the caudal lysis technique, but there are a few differences in technique and volumes of medication used.

The epidural space should be entered via the upper thoracic interspaces using a paramedian approach on the contralateral side. The most common levels are T1-2 and T2-3. Entry at these levels allows for a sufficient length of the catheter to remain in the epidural space after the target level has been reached. If the target is the lower cervical nerve roots, a more caudal interspace should be selected. We place the patient in the left lateral decubitus position, but use a prone approach in larger patients.

A technique referred to as the "3-D technique" is used to facilitate entry into the epidural space. The "3-D" stands for direction, depth, and direction. Using an AP fluoroscopic image, the initial direction of the 15-or 16-gauge RX Coudé needle is determined. Using a modified paramedian approach with the skin entry one and a half levels below the target interlaminar space, advance and direct the needle toward the midpoint of the chosen interlaminar space with the opening of the needle pointing medial. Once the needle engages the deeper tissue planes (usually at 2 to $3 \mathrm{~cm}$ ), check the depth of the needle with a lateral image. Advance the needle toward the epidural space and check repeat images to confirm the depth. The posterior border of the dorsal epidural space can be visualized by identifying the junction of the base of the spinous process of the vertebra with its lamina. This junction creates a distinct radiopaque "straight line." Once the needle is close to the epidural space, obtain an AP fluoroscopic image to recheck the direction of the needle. If the tip of the needle has crossed the midline as defined by the spinous processes of the vertebral bodies, pull the needle back and redirect. The " 3 -D" process can be repeated as many times as is necessary to get the needle into the perfect position.

Using loss-of-resistance technique, advance the needle into the epidural space with the tip of the RX-Coudé needle, pointed caudally. Once the tip is in the epidural space, rotate the tip cephalad, and inject 1 to $2 \mathrm{~mL}$ of contrast to confirm entry. Rotation or movement of any needle in the epidural space can cut the dura. This technique has been improved with the advent of the RX Coudé 2 needle, which has a second interlocking stylet that protrudes slightly beyond the tip of the needle and functions to push the dura away from the needle tip as it is turned 180 degrees cephalad (Figures 28-32). 


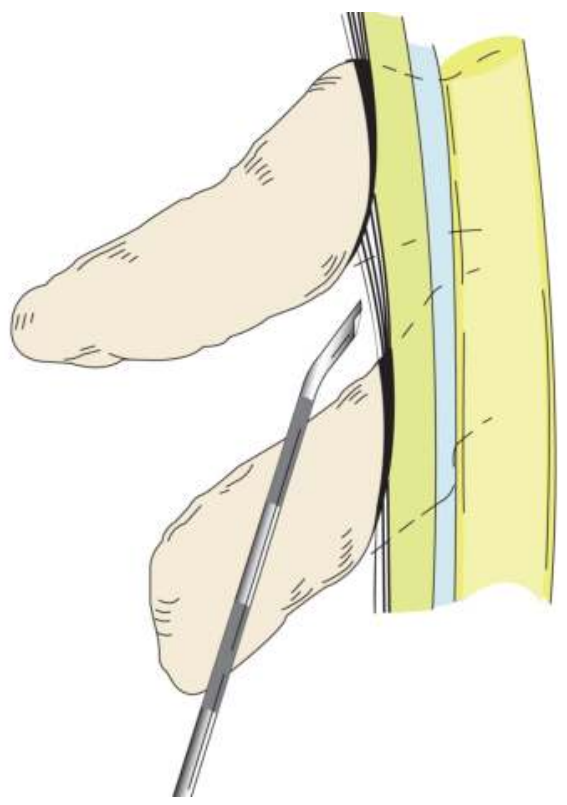

Figure 28. Sequence of stages to place a catheter using the R-X Coude, part 1: The needle is inserted into the epidural space with the tip directed as shown.

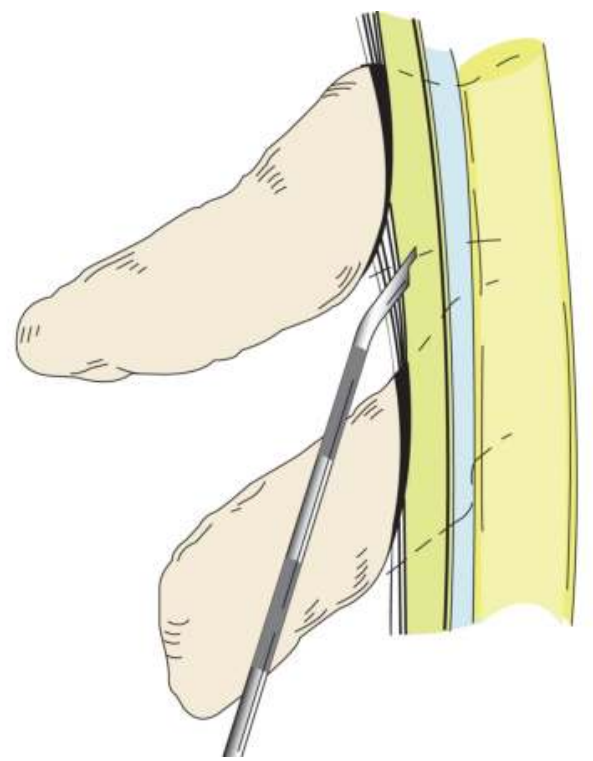

Figure 29. Sequence of stages to place a catheter using the R-X Coude, part 2: The needle is inserted into the epidural space with the tip directed as shown. 


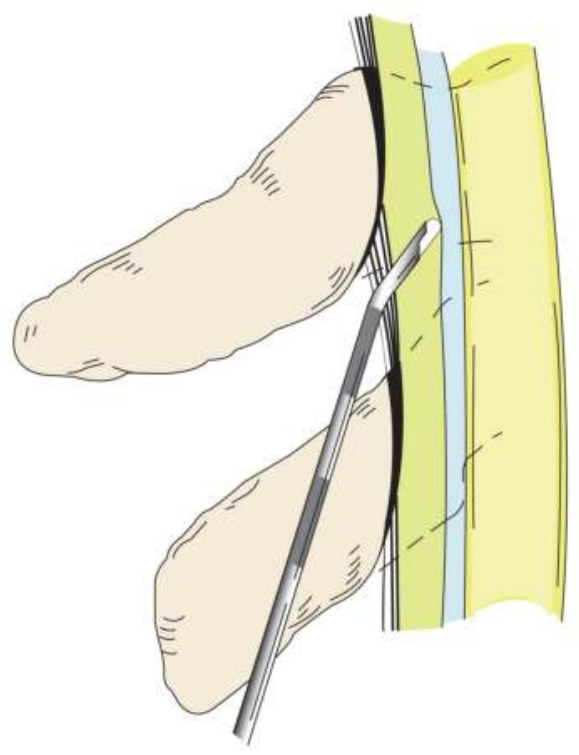

Figure 30. Sequence of stages to place a catheter using the R-X Coude, part 3: The protruding stylet is inserted.

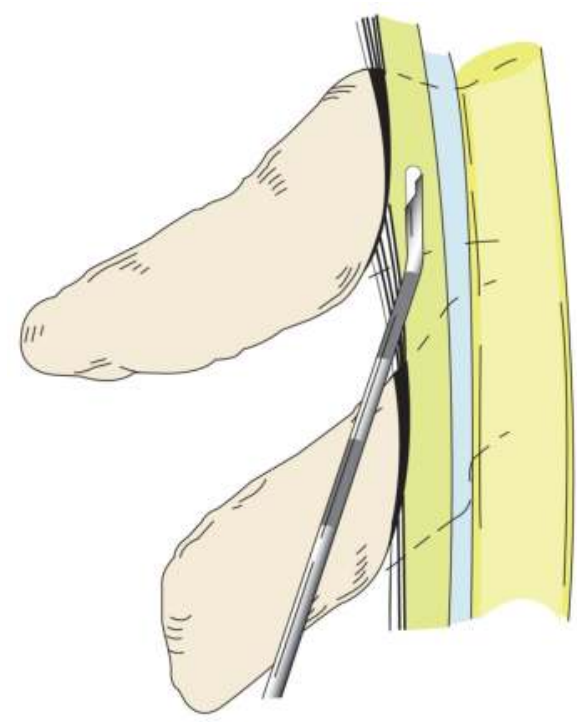

Figure 31. Sequence of stages to place a catheter using the R-X Coude, part 4: Then the needle is rotated so the tip is parallel to the dura. 


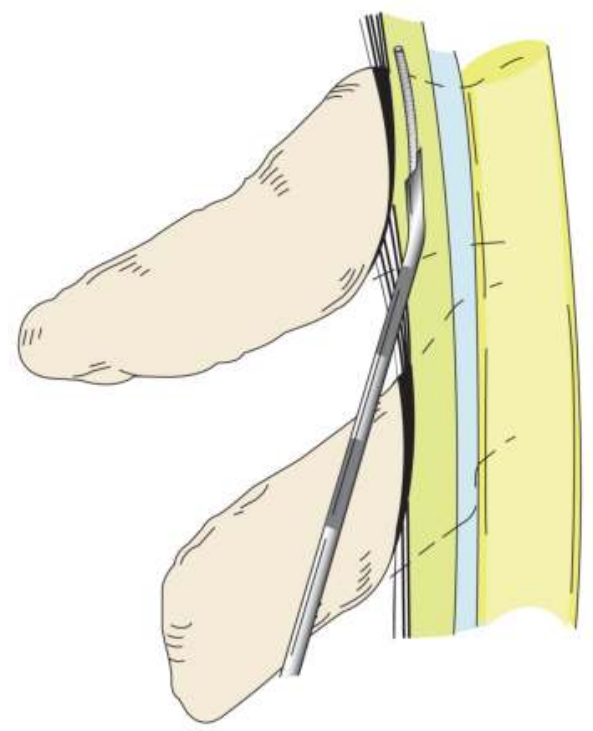

Figure 32. Sequence of stages to place a catheter using the R-X Coude, part 5: The catheter is inserted.

Inject an additional small volume as needed to complete the epidurogram. If there is no free flow of injected contrast, pressure may build up in the lateral epidural space. Characteristic fluid spread by the path of least resistance can be recognized as perivenous counter spread (PVCS). Presence of PVCS means pressure builds up in the lateral epidural space that is unable to spread laterally to decompress. The dye spread picks the path of least resistance to the opposite side. Pressure may build up and lead to ischemic spinal cord injury. Flexion and rotation of the head and neck can open up lateral runoff and release the pressure through the enlarged neural foramina (Figure 33) [38].

As with the caudal epidurogram, look for filling defects. It is extremely important to visualize spread of the contrast in the cephalad and caudal directions. Loculation of contrast in a small area must be avoided as this can significantly increase the pressure in the epidural space and can compromise the already tenuous arterial blood supply to the spinal cord. Place a bend on the catheter as previously described for the caudal approach and insert it through the needle (Figure 32). The opening of the needle should be directed toward the target side. Slowly advance the catheter to the lateral gutter and direct it cephalad. Redirect the catheter as needed and once the target level has been reached, turn the tip of the catheter toward the foramen (Figure 34). Inject 0.5 to $1 \mathrm{~mL}$ of contrast to visualize the target nerve root. Make sure there is runoff of contrast out of the foramen (Figure 35). Slowly instill $150 \mathrm{U}$ of Hylenex dissolved in $5 \mathrm{~mL}$ of preservative-free normal saline. Follow this with 1 to $2 \mathrm{~mL}$ of additional contrast and observe for "opening up" of the "scarred in" nerve root. Give a $2 \mathrm{~mL}$ test dose of a $6 \mathrm{~mL}$ solution of LA/ S. Our combination is $5 \mathrm{~mL}$ of $0.2 \%$ ropivicaine and $4 \mathrm{mg}$ of dexamethasone. If after 5 minutes there is no evidence of intrathecal or intravascular spread, inject the remaining $4 \mathrm{~mL}$. Remove 


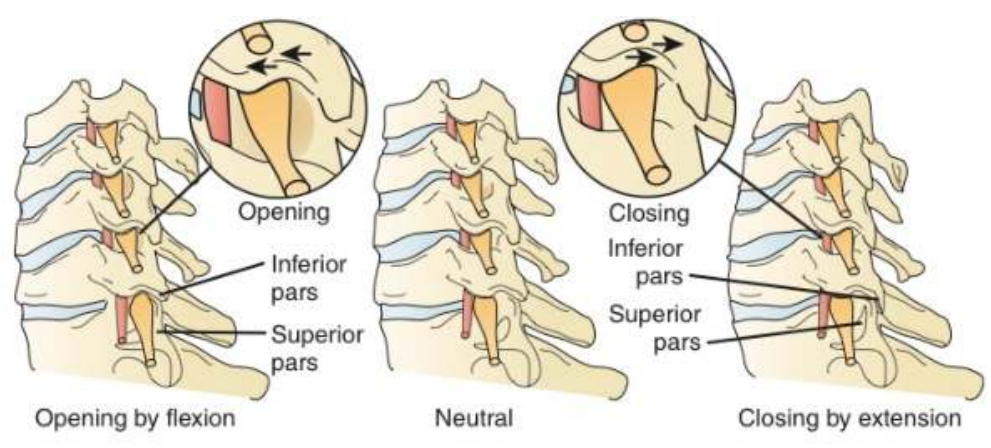

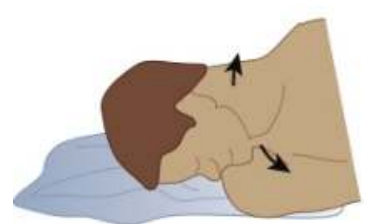

Opening

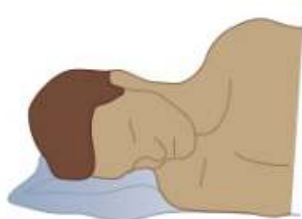

Neutral

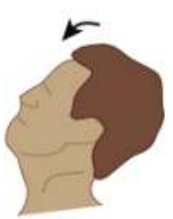

Closing

Figure 33. Flexion rotation, left to right regardless patient position. The neural foramen enlarges on flexion rotation and gets smaller with extension. The inferior pars slides forward over the superior pars to enlarge the foramen. This allows lateral run off and pressure release with PVCS.

the needle, and secure and dress the catheter as previously described. Once 20 minutes have passed since the last dose of LA/S solution and there is no evidence of a subarachnoid or subdural block, start an infusion of $5 \mathrm{~mL}$ of hypertonic saline over 30 minutes. At the end of the infusion, flush the catheter with 1 to $2 \mathrm{~mL}$ of preservative-free normal saline and cap the catheter.

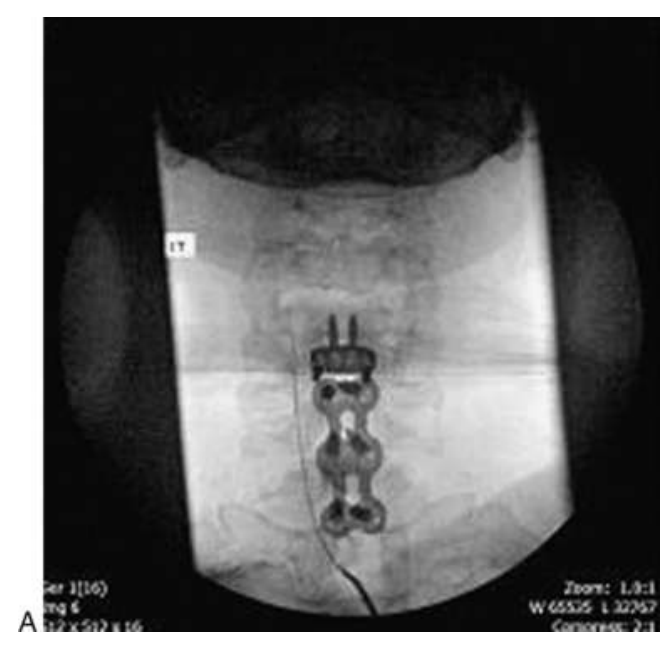

Figure 34. Cervical left ventral lateral catheter to the upper level of fusion C5-7. 


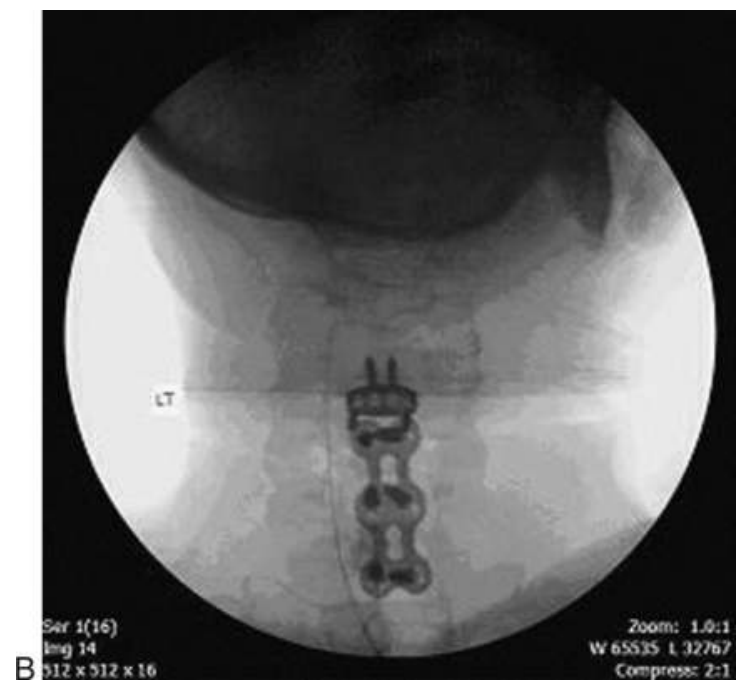

Figure 35. Cervical-left ventral lateral catheter threaded to above level of fusion of C4. The dye injection spreads cephalad and lateral.

The second and third infusions are performed on the next day with $6 \mathrm{~mL}$ of $0.2 \%$ ropivacaine without spread and $5 \mathrm{~mL}$ of hypertonic saline using the same technique and precautions described for the first infusion. The catheter is removed and prophylactic antibiotics are prescribed. Clinic follow-up is 30 days.

\section{Thoracic lysis of adhesions}

The technique for entry into the thoracic epidural space for adhesiolysis is identical to that for the cervical region. Always remember the 3-D technique. Make sure to get a true lateral when checking the depth of the needle. This can be obtained by superimposing the rib shadows on one another. The target is still the ventrolateral epidural space with the tip of the catheter in the foramen of the desired level. The major difference for thoracic lysis compared to the caudal and cervical techniques is the volumes of the various injectates. Volumes of $8 \mathrm{~mL}$ are used for the contrast, Hylenex, LA/S, and hypertonic saline. Table 1 lists typical infusion volumes for epidural adhesiolysis. 


\begin{tabular}{lllll}
\hline Contrast & $\begin{array}{l}\text { Hyaluronidase and } \\
\text { Normal Saline }\end{array}$ & $\begin{array}{l}\text { Local Anesthetic and } \\
\text { Steriod }\end{array}$ & $\begin{array}{l}\mathbf{1 0 \%} \text { Hypertonic Saline } \\
\text { Infusion }\end{array}$ \\
\hline Caudal & $10 \mathrm{~mL}$ & $10 \mathrm{~mL}$ & $10 \mathrm{~mL}$ & $10 \mathrm{~mL}$ \\
\hline $\begin{array}{l}\text { Caudal and } \\
\text { transforaminal }\end{array}$ & $5 \mathrm{~mL}$ in each catheter & $5 \mathrm{~mL}$ in each catheter & $5 \mathrm{~mL}$ in each catheter & $\begin{array}{l}8 \mathrm{~mL} \text { in caudal catheter } \\
\text { and } 4 \mathrm{~mL} \text { in } \\
\text { transforaminal catheter }\end{array}$ \\
\hline $\begin{array}{l}\text { Thoracic } \\
\text { Cervical }\end{array}$ & $8 \mathrm{~mL}$ & $8 \mathrm{~mL}$ & $8 \mathrm{~mL}$ & $8 \mathrm{~mL}$ \\
\hline
\end{tabular}

Table 1. Typical Infusion Volumes for Epidural Adhesiolysis

\section{Neural flossing}

The protocol for epidural adhesiolysis has been aided by neural flossing exercises that were designed to mobilize nerve roots by "sliding" them in and out of the foramen (Figure 36). This breaks up weakened scar tissue from the procedure and prevents further scar tissue deposition. If these exercises are done effectively three to four times per day for a few months after the procedure, the formation of scar tissue will be severely restricted.

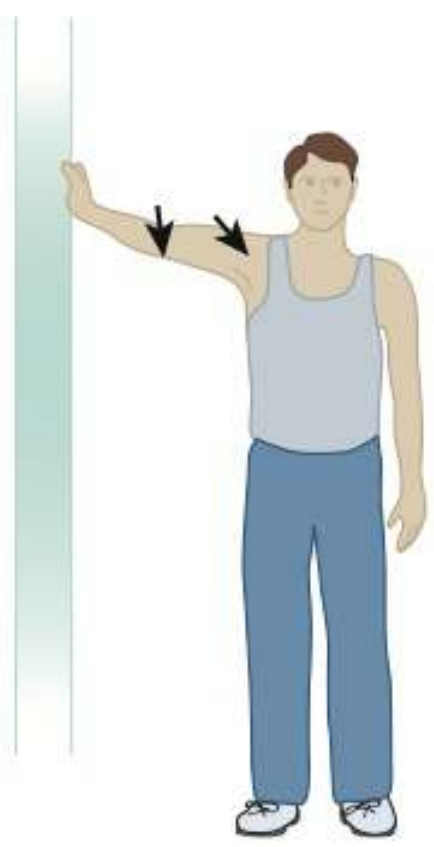

Figure 36. Neural flossing exercises, part 1: Standing erect, firmly grasp a stable surface (e.g., a door frame) with outstretched arm. Press elbow and shoulder forward. 


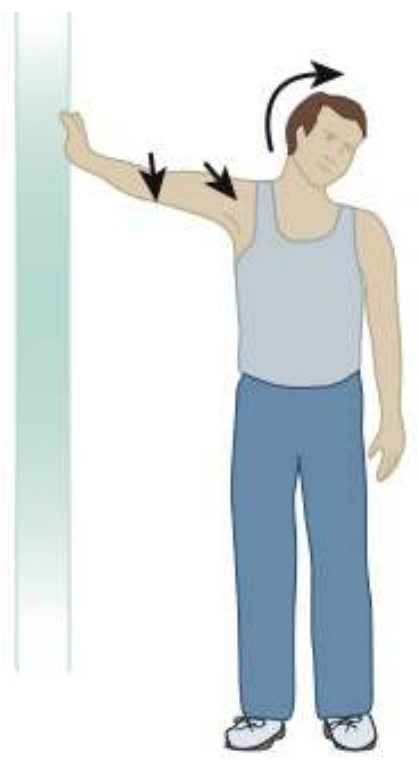

Figure 37. Neural flossing exercises, part 2: Next, slowly tilt head in opposite direction from outstretched arm to achieve gentle tension.

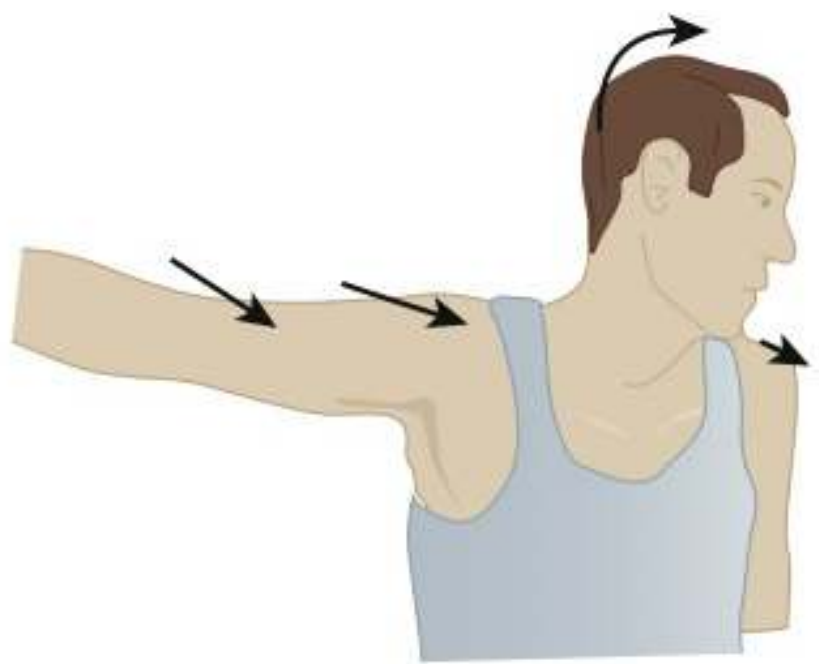

Figure 38. Neural flossing exercises, part 3: Finally, rotate chin toward opposite shoulder as is comfortable. Hold this final position for approximately 20 to 30 seconds. 


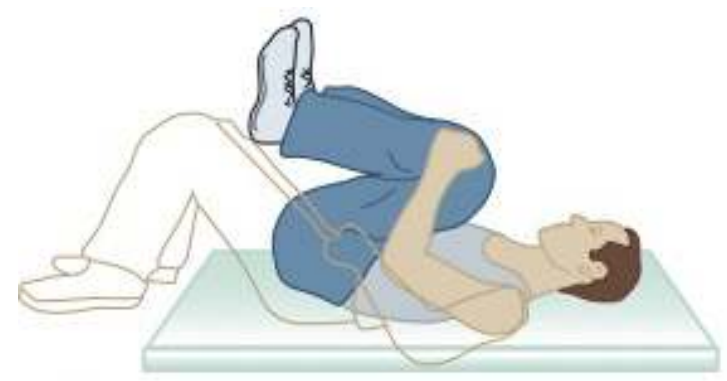

Figure 39. Neural flossing exercises, part 4: Lay down supine on an exercise mat without a pillow. Slowly bring both knees close to the chest with bent legs and hold this position for 20 seconds. Release and assume a neutral position.

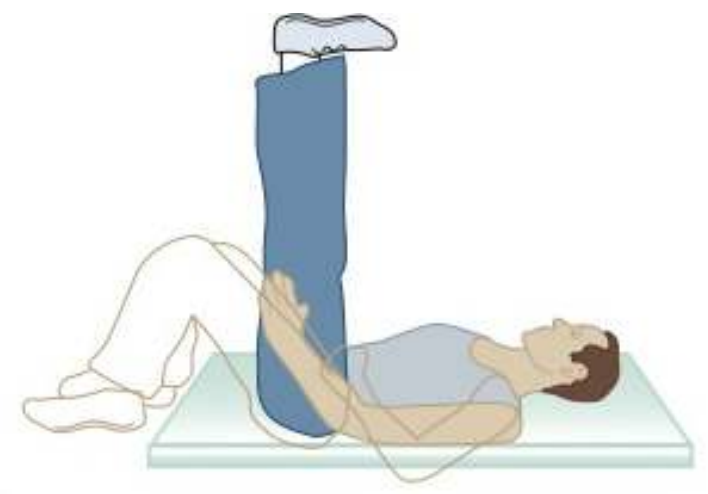

Figure 40. Neural flossing exercises, part 5: Again in supine position, raise both legs to 90 degrees, with knees straight while laying flat on a firm surface. Hold for 20 seconds. Assume a neutral position and rest briefly.

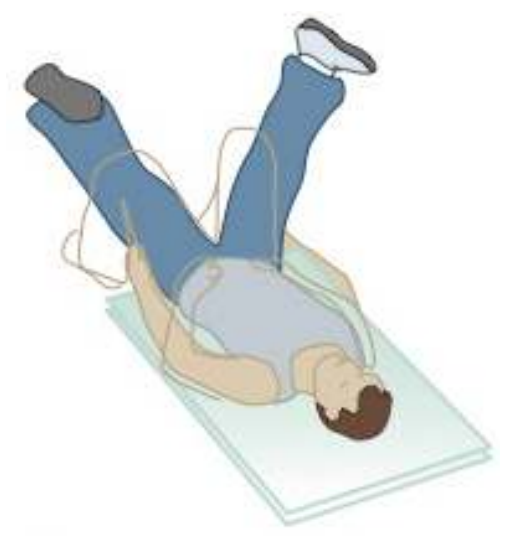

Figure 41. Neural flossing exercises, part 6: Bring both legs to a 90-degree angle while lying supine. Slowly spread legs in a $\mathrm{V}$ shape, as much as is comfortable, and hold for 20 seconds. 


\section{Epidural mapping}

In patients with multilevel radiculopathy and complex pain, it can be difficult to determine from where the majority of the pain is emanating. We have been using a technique that we have termed mapping to locate the most painful nerve root with stimulation and then carry out the adhesiolysis at that level. There are several references in the literature regarding the use of stimulation to confirm epidural placement of a catheter and for nerve root localization [39]. The TunL Kath and the TunL-XL catheter can be used as stimulating catheters to identify the nerve root(s).

After entering the epidural space, advance the catheter into the ventrolateral epidural space past the suspected target level. Make sure the tip of the catheter is pointing laterally toward the foramina, just below the pedicle. Pull the catheter stylet back approximately $1 \mathrm{~cm}$. Using alligator clips, attach the cathode to the stylet and ground the anode on the needle or ground pad or a 22-gauge needle inserted into the skin. Apply electrical stimulation with a stimulator box with a rate of 50 pulses per second and a pulse width of 450 milliseconds, dialing up the amplitude until a paresthesia is perceived in small increments, usually less than 2 or 3 volts. Inquire of the patient as to whether or not the paresthesia is felt in the area of the patient's recognized greatest pain. This process is repeated at each successive level until the most painful nerve root is identified. Once identified, the adhesiolysis is carried out at that level. The mapping procedure is also useful to identify the optimal site of surgery either before the first surgery or when surgery has failed one or more times.

\section{Complications}

As with any invasive procedure, complications are possible. These include bleeding, infection, headache, damage to nerves or blood vessels, catheter shearing, bowel/bladder dysfunction, paralysis, spinal cord compression from loculation of the injected fluids or hematoma, subdural or subarachnoid injection of local anesthetic or hypertonic saline, and reactions to the medications used. We also include on the consent form that the patient may experience an increase in pain or no pain relief at all.

Although the potential list of complications is long, the frequency of complications is very rare. However, there is clearly a learning curve, and recent studies reflect this by the significantly improved long-term outcome and the very rare publications of complications and medicolegal consequences when one considers the ever-increasing clinical experience.

Subdural spread is a complication that should always be watched for when injecting local anesthetic. During the caudal adhesiolysis, particularly if the catheter is advanced along the midline, subdural catheter placement is a risk (Figure 42 and 43). Identification of the subdural motor block should occur within 16 to 18 minutes. Catheters used for adhesiolysis should never be directed midline in the epidural space. 


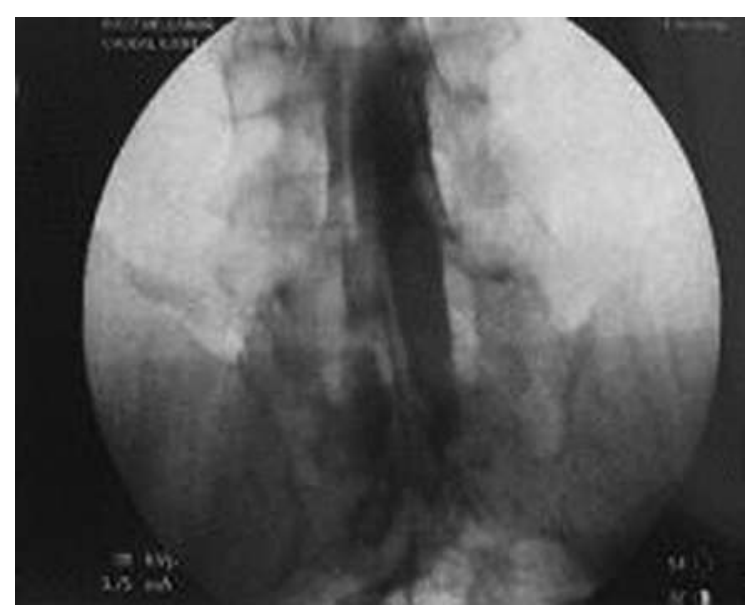

Figure 42. Midline catheter placement enters subdural space. There is also some epidural dye spread. But the patient starts to complain of bilateral leg pain.

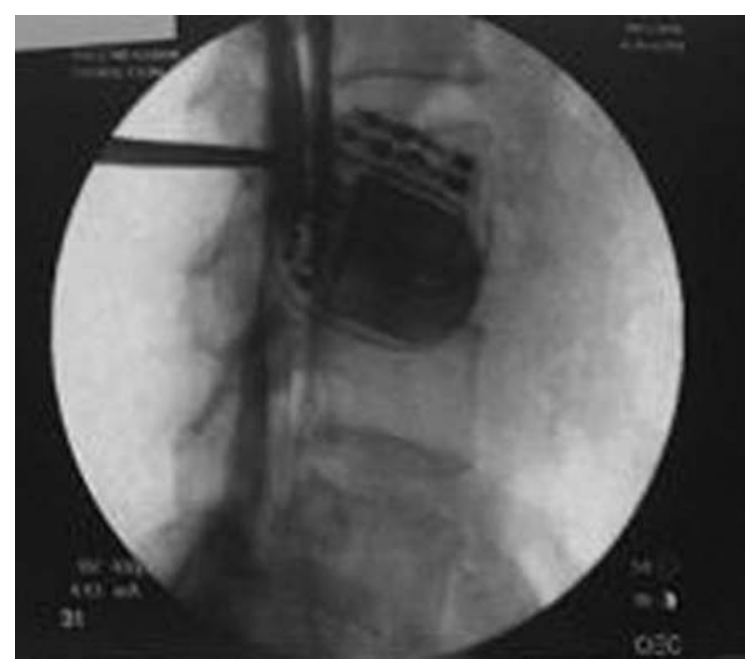

Figure 43. A 22-gauge spinal needle and extension set with syringe placed in the subdural space and $12 \mathrm{~mL}$ fluid aspirated. The patient reported immediate reversal of bilateral leg pain. Note the dye in the extension tubing and syringe at the 7-o'clock position.

Most hematomas and other major complications are associated with the use of sharp needles. The use of blunt needles or catheters should be considered to reduce the risk of major complications with the lysis procedure or transforaminal procedures [40].

Venous run off is most common on the first epidural procedure due to high-pressure veins being engorged and large. Following lysis of adhesions and fluid foraminotomy, these high- 
pressure veins are converted to low-pressure veins and venous run off is less likely. In fact, no cases of hematoma have been reported after lysis of adhesions and fluid foraminotmy in the ventrolateral epidural space [41].

A case of a hematoma has been reported after the MILD procedure before lysis was performed. Lysis should be considered prior to the MILD procedure to achieve fluid foraminotomies and allow fluid to pass out of the spinal canal and avoid venous run off and hematomas [42].

\section{Outcomes}

Initially in the early 1980s the protocol was designed to direct site-specific medication onto the dorsal root ganglion; however, after performing a number of the procedures, it was found that the dorsal root ganglion was exceptionally hard to reach secondary to developing scar tissue or adhesions. In the early days, our understanding was coming from the use of local anesthetics for surgery giving a 2-to 4-hour block for the surgeon to operate. It was gratifying to see chronic pain patients get months and years of pain relief following the placement of the new steerable x-ray visible catheter. The early report in 1985 by Racz et al [43] described the use of phenol at the dorsal root ganglion followed by an observational listing of outcomes that were clearly not as good as the latest studies on failed back surgery and spinal stenosis showing $75 \%$ to $80 \%$ improvement at 12 months' follow-up by Manchikanti [38]. Initially we were pleased to see some patients getting 3 to 4 months of relief and report seeing recovery of footdrops. This philosophy still proves to be true even in studies in 2008 by Sakai et al [44] in which they found that adhesiolysis with catheter-directed steroid and local anesthetic injection during epiduroscopy alleviated pain and reduced sensory nerve dysfunction in patients with chronic sciatica. The evolution of these findings has changed the process into what it is today [45]. Racz and Holubec first reported on epidural adhesiolysis in 1989 [46]. There were slight variations in the protocol compared to today's protocol, namely the dose of local anesthetic and the fact that hyaluronidase was not used. Catheter placement was lesion-specific (i.e., the tip of the catheter was placed in the foramen corresponding to the vertebral level and side of the suspected adhesions). The retrospective analysis conducted 6 to 12 months after the procedure reported initial pain relief in $72.2 \%$ of patients $(N=72)$ at time of discharge. Relief was sustained in $37.5 \%$ and $30.5 \%$ of patients at 1 and 3 months, respectively. Forty-three percent decreased their frequency and dosage of medication use and $16.7 \%$ discontinued their medications altogether. In total, $30.6 \%$ of patients returned to work or returned to daily functions. In April 1990, at a presentation of the 7th IASP World Congress on Pain in Adelaide, Austraila, Arthur et al [47] reported on epidural adhesiolysis in 100 patients, 50 of whom received hyaluronidase as part of the procedure. In the hyaluronidase group, $81.6 \%$ of the participants had initial pain relief, with $12.3 \%$ having persistent relief; $68 \%$ of the no hyaluronidase group had relief of pain, with $14 \%$ having persistent relief at the end of the 3-year follow-up period from which the study sample was randomly selected.

An informal survey of ophthalmologic anesthesiologists found no cases of anaphylaxis to hyaluronidase used for retrobulbar blocks. In this survey, skin testing for allergy to hyaluronidase was not reported. This implies that severe allergic reactions are rare; however, it is 
recommended that these procedures be performed in an environment with resuscitative equipment [48].

In 1994 Stolker et al [49] added hyaluronidase to the procedure, but omitted the hypertonic saline. In a study of 28 patients, they reported greater than $50 \%$ pain reduction in $64 \%$ of patients at 1 year. They stressed the importance of the patient selection and believed that the effectiveness of adhesiolysis was based on the effect of the hyaluronidase on the adhesions and the action of the local anesthetic and steroids on the sinuvertebral nerve.

Devulder et al published a study of 34 patients with failed back surgery syndrome in whom epidural fibrosis was suspected or proved with MRI [50] and an epidural catheter was inserted via the sacral hiatus to a distance of $10 \mathrm{~cm}$ into the caudal canal. Injections of contrast dye, local anesthetic, corticosteroid, and hypertonic saline (10\%) were carried out daily for 3 days. No hyaluronidase was used. Filling defects were noted in 30 of 34 patients, but significant pain relief was noted in only 7 patients at 1 month, 2 patients at 3 months, and no patients at 12 months. They concluded that epidurography may confirm epidural filling defects for contrast dye in patients with filling defects, but a better contrast dye spread, assuming scar lysis does not guarantee sustained pain relief. This study was criticized for lack of lesion-specific catheter placement resulting in nonspecific drug delivery [51]. The catheter was never directed to the ventral lateral epidural space where the dorsal root ganglion is located and the lateral recess scarring occurs.

Heavner et al [52] performed a prospective randomized trial of lesion-specific epidural adhesiolysis on 59 patients with chronic intractable low back pain. The patients were assigned to one of four epidural adhesiolysis treatment groups: (1) hypertonic (10\%) saline plus hyaluronidase, (2) hypertonic saline, (3) isotonic (0.9\%) saline, or (4) isotonic saline plus hyaluronidase. All treatment groups received corticosteroid and local anesthetic. Overall, across all four treatment groups, 83\% of patients had significant pain relief at 1 month compared to $49 \%$ at 3 months, $43 \%$ at 6 months, and $49 \%$ at 12 months. The hyaluronidase and the hypertonic saline study group had a much lower incidence of additional need for pain procedures than the placebo groups, showing that site-specific catheter placement is important. Active substances and preservative free normal saline were blinded for the placebo effect.

Manchikanti et al [53] performed a retrospective randomized evaluation of a modified Racz adhesiolysis protocol in 232 patients with low back pain. The study involved lesion specific catheter placement, but the usual 3-day procedure was reduced to a 2-day (group 1) or a 1-day (group 2) procedure. Group 1 had 103 patients and group 2 had 129 patients. Other changes included changing the local anesthetic from bupivacaine to lidocaine, substituting methylprednisolone acetate or betamethasone acetate and phosphate for triamcinolone diacetate, and reduction of the volume of injectate. Of the patients in groups 1 and 2,62\% and $58 \%$ had greater than $50 \%$ pain relief at 1 month, respectively, with these percentages decreasing to $22 \%$ and $11 \%$ at 3 months, $8 \%$ and $7 \%$ at 6 months, and $2 \%$ and $3 \%$ at 1 year. Of significant interest is that the percentage of patients receiving greater than $50 \%$ pain relief after four procedures increased to $79 \%$ and $90 \%$ at 1 month, $50 \%$ and $36 \%$ at 3 months, $29 \%$ and $19 \%$ at 6 months, and $7 \%$ and $8 \%$ at 1 year for groups 1 and 2, respectively. Short-term relief of pain was demonstrated, but long-term relief was not. 
Manchikanti, in 1999, evaluated two groups of randomly pulled, 150 patients for a 2-day reinjection procedure, and a second 150 patients for a one-day procedure out of a pool of 536 patients. It was concluded that repeat use of the one-day procedure is also cost effective when evaluated on a 12-month follow-up. The cost effectiveness indicated the lysis procedure to be superior to surgery or the rehabilitation activity program [53].

In a randomized, prospective study, Manchikanti et al [54] evaluated a 1-day epidural adhesiolysis procedure against a control group of patients who received conservative therapy. Results showed that cumulative relief, defined as relief greater than $50 \%$ with one to three injections, in the treatment group was $97 \%$ at 3 months, $93 \%$ at 6 months, and $47 \%$ at 1 year. The study also showed that overall health status improved significantly in the adhesiolysis group. Conservative therapy consisted of physical therapy and medications.

In 2004 Manchikanti et al [55] published their results of a randomized, double-blinded, controlled study on the effectiveness of 1-day lumbar adhesiolysis and hypertonic saline neurolysis in treatment of chronic low back pain. Seventy-five patients whose pain was unresponsive to conservative modalities were randomized into one of three treatment groups. Group 1 (control group) underwent catheterization where the catheter was in the sacral canal without adhesiolysis, followed by injection of local anesthetic, normal saline, and steroid. Group 2 consisted of catheterization with site-specific catheter placement being ventral-lateral for adhesiolysis, followed by injection of local anesthetic, normal saline, and steroid. Group 3 consisted of site-specific catheter placement for adhesiolysis, followed by injection of local anesthetic, hypertonic saline, and steroid. Patients were allowed to have additional injections based on the response, either after unblinding or without unblinding after 3 months. Patients without unblinding were offered either the assigned treatment or another treatment based on their response. If the patients in group 1 or 2 received adhesiolysis and injection and injection of hypertonic saline, they were considered withdrawn, and no subsequent data were collected. Outcomes were assessed at 3, 6, and 12 months using visual analog scale pain scores, Oswestry Disability Index, opioid intake, range-of-motion measurement, and P-3. Significant pain relief was defined as average relief of $50 \%$ or greater. Seventy-two percent of patients in group 3 , $60 \%$ of patients in group 2 , and $0 \%$ of patients in group 1 showed significant pain relief at 12 months. The average number of treatments for 1 year was 2.76 in group 2 and 2.16 in group 3 . Duration of significant relief with the first procedure was $2.8+1.49$ months and 3.8+3.37 months in groups 2 and 3, respectively. Significant pain relief $(>50 \%)$ was also associated with improvement in Oswestry Disability Index, range of motion, and psychologic status.

Manchikanti et al $[56,57]$ furthered this research using comparisons of percutaneous adhesiolysis versus fluoroscopically guided caudal epidural steroid injections. The first study involved a population of patients with chronic low back pain and known spinal stenosis. The results showed a $76 \%$ reduction in pain relief at 1 year with epidural adhesiolysis compared to $4 \%$ in the control group. The second study performed in a population of patients with postlumbar surgery syndrome showed a reduction in pain and improvement in functional status in $73 \%$ of the epidural adhesiolysis group compared to $12 \%$ in the control group.

In 2006 a study by Veihelmann et al [58] evaluated patients with a history of chronic low back pain and sciatica. Inclusion criteria were radicular pain with a corresponding nerve root 
compressing substrate found on MRI or CT. All patients were randomized to receive physiotherapy, analgesics, or lysis of adhesions. The lysis group had statistically significantly better outcome than the physical therapy treatment group.

Two other prospective evaluations by Chopra et al and Gerdesmeyer et al [59,60] evaluated patients with monosegmental radiculopathy of the lumbar spine. All the patients suffered from chronic disk herniations or failed back syndrome. All these randomized trials showed positive short-term and long-term relief. Two prospective evaluations also showed positive short-and long-term relief $[60,61]$.

Gerdesmeyer has published a prospective double blind placebo controlled multicenter trial, which has been the most significant evaluation of the technique. The target site remained ventral lateral at the most likely level of the pain generator. The study continued for over 12 months and the significant finding was that the study arm of the procedure showed better outcome at all points of measurements. The placebo arm was a subcutaneously placed catheter so that the patient could not tell the difference during the three daily reinjections or subsequently. The study has succeeded in differentiating the placebo group from the treatment group in each location. The results have led to the conclusion that percutaneous lysis of adhesions for patients with chronic lumbosacral radicular pain should be offered this procedure as first choice of treatment [62].

A systematic review of percutaneous adhesiolysis for chronic low back pain in post lumbar surgery syndrome and spinal stenosis by S Helm II, et al, found effectiveness of the procedure in both spinal stenosis and in post-lumbar surgery syndrome [59]. Additionally it was noted that there have not been any hematomas reported. The results of the review support the use of the procedure for the conditions listed.

The randomized double blind active control trial by Koh, et al, in patients with lateral spinal canal stenosis demonstrated that the hypertonic saline showed significant short-term pain relief [63]. Post procedure pain after the use of steroids has been a significant problem at the first recognition of the effectiveness of percutaneous lysis of adhesions. Patients reported significant post procedural pain prior to the introduction of hyaluronidase and hypertonic saline to the sequence of injections. The parallel observation from the use of increased volume of injection was that the hypertonic saline addition has not only reduced the radiculopathy pain but also reduced the patient's back pain. The volume increase was from the $2 \mathrm{~mL}$ per injection range to the $5 \mathrm{~mL}$ range of each fluid component. The sequence of injections is first contrast, followed by hyaluronidase, local anesthetic and steroid, and 20-30 minutes later, if there was no motor block, the injection of hypertonic saline.

Manchikanti's, et al, two-year follow-up of randomized controlled trial compared one-day lysis of adhesions procedure to caudal epidural injection where the reinjection was triggered by the patient's pain relief dropping to below 50\%. During the two-year study, the study group received $6.4 \pm 2.35$ procedures and $82 \%$ of the patients received at least $50 \%$ pain relief, whereas the caudal epidural injection had $5 \%$ similar rating. This strongly supports the effectiveness of the percutaneous epidural lysis of adhesions [64]. 
Park's, et al, evaluation of severity of spinal stenosis with transforaminal adhesiolysis and lumbar neuroforaminal stenosis showed effectiveness regardless of the intensity of lumbar stenosis [65].

Park, et al, evaluated epidural neuroplasty for cervical disc herniation and demonstrated effectiveness when conservative measures had failed. Park, et al, evaluated epidural neuroplasty for cervical disc herniation and found safety and efficacy. There was no control arm to the study, but the clinical results indicate reduction in cervical radiculopathy. The overall clinical experience has showed us that there is a need for evaluation for cervicogenic facet pain and appropriate treatment. Additionally, the anterior compartment between the anterior and middle scalene muscles may be additional pain generators in patients that have pain secondary to facet joint arthropathy [66].

Choi, et al, compared two patient groups with herniation of intervertebral discs and post lumbar surgery syndrome. These results indicate better outcome in non-operated patients. While not absolute prognostic predictor, the recommendation is that percutaneous adhesiolysis is a reasonable non-operative treatment option of herniation of intervertebral discs, spinal stenosis, and post lumbar surgery syndrome [67].

The cost effectiveness of the Racz procedure compared favorably to other treatments for the same conditions. The cost utility for 1 year of quality-adjusted life year (QALY) of USD is $\$ 2,652$ for post-lumbar surgery syndrome and USD \$2,649 for lumbar central spinal stenosis [68].

Epidural adhesiolysis has evolved over the years as an important treatment option for patients with intractable cervical, thoracic, and low back and leg pain. Studies show that patients are able to experience significant pain relief and restoration of function. Manchikanti's studies show that the amount and duration of relief can be achieved by repeat procedures. Recent prospective randomized double-blind studies on failed back surgery and spinal stenosis show $75 \%$ and $80 \%$ improvement in visual analog scale scores and functional improvements at 12 months' follow-up. There have been no negative studies to date where the lysis target was the ventral-lateral epidural space. The one negative study used a $10 \mathrm{~cm}$ sacral mid-canal catheter placement which was non-target specific [51]. This negative study was subsequently used as the placebo group in a study performed by Manchikanti. Manchikanti's study consisted of 3 treatment groups: placebo (sacral mid-canal catheter placement), target specific ventral-lateral epidural without hypertonic saline and target specific ventral-lateral epidural with hypertonic saline. The later two treatment groups had positive outcomes with the hypertonic saline group superior, whereas, the placebo group did not [55]. The evolution in the recognition of the sitespecific importance of the catheter and medication delivery together with the fact that physicians need to acquire the skills to be able to carry out the procedure led to the improved outcomes seen in recent prospective randomized studies.

The management of failed back surgery syndrome and post laminectomy syndrome will likely continue to be controversial among the multitude of practitioners who treat these patients. However, in experienced hands, it is established as a reasonable option for many patients.

Percutaneous neuroplasty via a transforaminal approach evolved from the caudal approach. Lysis of adhesions via the caudal approach involves introducing a catheter through the sacral 
hiatus and advancing it to the affected nerve root in the ventral-lateral epidural space. On the other hand, transforaminal percutaneous neuroplasty achieves a midline catheter placement in the epidural space that is able to target the two most heavily innervated structures in the spine-the posterior annulus fibrosus and the posterior longitudinal ligament [5]. Apart from a surgical approach, the ventral epidural structures have been otherwise inaccessible.

Endoscopy offers direct visualization of the affected nerve roots in addition to mechanical adhesiolysis, and may become more mainstream as the technique is refined.

Facet pain is commonly associated with the postlysis period or after provocative testing a month or so later if two-facet diagnostic blocks show efficacy. In addition to epidural lysis of adhesions, the combined use of radiofrequency facet denervation gives us the best long-term outcome.

Epidural adhesiolysis has been accepted as a treatment for post laminectomy syndrome, failed back syndrome, and cervical and thoracic radicular syndromes. Additional studies are underway to further refine the technique and indications. The combined use of long term patient education for neural flossing exercises and the inclusion of the facet delayed treatment in the algorithm further improves patient outcome. The identification of back pain provocation by saline injection and the successful use of percutaneous neuroplasty in the treatment represents hopeful promise for a cost effective treatment of back pain.

The increasing overall evidence is positive in the recommendation for use of percutaneous lysis of adhesions based on high quality and observational clinical studies. The procedure recommendation is for patients that failed conservative therapies. There are no negative studies reported regarding the use of percutaneous adhesiolysis from the sacral to the cervical areas.

The diagnosis and treatment of unusual rare complications must be within the scope of the physician's practice and the postoperative observational periods. Delayed secondary motor block in patients where only caudal catheter is used to treat spinal stenosis needs to be recognized as a consequence of fluid expansion from osmotic effect. Our preferred clinical practice is heading in the direction of caudal and transforaminal catheter use at the level of stenosis based on the utilization of the above-mentioned transforaminal catheter reports [4].

Clearly, additional studies will further prove safety and efficacy. Rare problems will come to light, such as allergies, unusual loculations, or syrinx or congenital malformations. Thus, the field shall become similar to any other advanced medical intervention. The quality of outcome improves with improved training and experience. The most significant hazard is physicians that are not trained, claiming to carry out percutaneous lysis procedure without appropriate catheter placement. Therefore, recommendation is to describe the procedure and/or save procedure fluoroscopic images that will prove appropriate catheter placement on anteriorposterior and lateral views. Midline catheter placement for lysis of adhesions should be avoided.

The treatment algorhythm for patients with leg and back pain, based on accumulating evidence, should focus on radiculopathy and back pain. Next, a month later, the patient must 
be examined for diagnosis and treatment of other causes of back pain, such as facet joint related, and pain from muscle spasms like gluteus medius, para spinal, quadratus lumborum, psoas, and piriformis muscle related radiculopathy in the lumbosacral area. Significant undiagnosed problems include trochanteric bursa related pain, cluneal nerve entrapments and hip joint arthropathies. Similarly, the order of evaluation and treatment in the upper extremity addressed should begin with radiculopathy, followed by facet joints and interscalene entrapments. Involvement through neural flossing exercises and appropriate instructions as outlined in the above text has been remarkably well accepted by the patients.

\section{Acknowledgements}

Racz GB, Day MR, Heavner JE, Scott J. Lysis of Epidural Adhesions. In: Waldman S, ed. Pain Management, 2 ${ }^{\text {nd }}$ Edition. Elsevier; 2011: 1258-1272.

Racz GB, Day MR, Heavner JE, Smith JP. The Racz Procedure: Lysis of Epidural Adhesions (Percutaneous Neuroplasty). In: Deer T, ed. The AAPM Text of Pain Medicine. Springer; 2011.

The authors would also like to thank Marzieh N. Brown and Paula Brashear, Katie Clower and Floramay Holidayfor their assistance in the editing of this chapter.

\section{Author details}

Gabor B. Racz ${ }^{1}$, James E. Heavner ${ }^{1}$, Jeffrey P. Smith ${ }^{1}$, Carl E. Noe², Adnan Al-Kaisy³, Tomikichi Matsumoto ${ }^{4}$, Sang Chul Lee ${ }^{5}$ and Laszlo Nagy ${ }^{6}$

*Address all correspondence to: gbracz@yahoo.com

1 Texas Tech University Health Sciences Center, Lubbock, Texas, USA

2 University of Texas Southwestern Medical Center, Dallas, Texas, USA

3 London Bridge Hospital, London, UK

4 Shiga University of Medical Science, Shiga, Japan

5 Department of Anesthesiology and Pain Medicine, Seoul National University Hospital, Seoul, Korea

6 Texas Tech University Health Sciences Center and Covenant Medical Center, Department of Pediatric Neurosurgery, USA 


\section{References}

[1] Lawrence R., Helmick C., Arnett F., et al: Estimates of the prevalence of arthritis and selected musculoskeletal disorders in the United States. Arthritis Rheum 1998; 41(5): 778-799.

[2] Straus B.: Chronic pain of spinal origin: the costs of intervention. Spine 2002; 27(22): 2614-2619.

[3] National Center for Health Statistics : National hospital discharge survey, Washington, DC, US Department of Health and Human Services, Centers for Disease Control and Prevention, 1990. Report no. PB92-500818

[4] Van Zundert J.: Personal communication. 2005.

[5] Kuslich S., Ulstrom C., Michael C.: The tissue origin of low back pain and sciatica. Orthop Clin North Am 1991; 22:181-187.

[6] Racz G., Noe C., Heavner J.: Selective spinal injections for lower back pain. Curr Rev Pain 1999; 3:333-341.

[7] Anderson S.: A rationale for the treatment algorithm of failed back surgery syndrome. Curr Rev Pain 2000; 4:396-406.

[8] Pawl R.: Arachnoiditis and epidural fibrosis: the relationship to chronic pain. Curr Rev Pain 1998; 2:93-99.

[9] Cervellini P., Curri D., Volpin L., et al: Computed tomography of epidural fibrosis after discectomy: a comparison between symptomatic and asymptomatic patients. Neurosurgery 1988; 23(6):710-713.

[10] Manchikanti L., Staats P., Singh V.: Evidence-based practice guidelines for interventional techniques in the management of chronic spinal pain. Pain Phys 2003; 6:3-81.

[11] LaRocca H., Macnab I.: The laminectomy membrane: studies in its evolution, characteristics, effects and prophylaxis in dogs. J Bone Joint Surg 1974; 5613:545-550.

[12] Cooper R., Freemont A., Hoyland J., et al: Herniated intervertebral disc-associated periradicular fibrosis and vascular abnormalities occur without inflammatory cell infiltration. Spine 1995; 20:591-598.

[13] McCarron R., Wimpee M., Hudkins P., et al: The inflammatory effects of nucleus pulposus; a possible element in the pathogenesis of low back pain. Spine 1987; 12:760-764.

[14] Parke W., Watanabe R.: Adhesions of the ventral lumbar dura: an adjunct source of discogenic pain?. Spine 1990; 15:300-303.

[15] Viesca C., Racz G., Day M.: Special techniques in pain management: lysis of adhesions. Anesthesiol Clin North Am 2003; 21:745-766. 
[16] Songer M., Ghosh L., Spencer D.: Effects of sodium hyaluronate on peridural fibrosis after lumber laminectomy and discectomy. Spine 1990; 15:550-554.

[17] Key J., Ford L.: Experimental intervertebral disc lesions. J Bone Joint Surg Am 1948; 30:621-630.

[18] Olmarker K., Rydevik B.: Pathophysiology of sciatica. Orthop Clin North Am 1991; 22:223-233.

[19] Ross J., Robertson J., Frederickson R., et al: Association between peridural scar and recurrent radicular pain after lumbar discectomy; magnetic resonance evaluation. Neurosurgery 1996; 38:855-863.

[20] Gilbert K., Brismee J., Collins D., et al: Lumbosacral nerve roots displacements and strain: part 1. A novel measurement technique during straight leg raise in unembalmed calavers. Spine 2007; 32(14):1513-1520.Phila Pa 1976

[21] Heavner JE, Chokhavatia S, Kizelshteyn G: Percutaneous evaluation of the epidural and subarachnoid space with a flexible fiberscope, Reg Anesth 1991;15:85.

[22] Bosscher HA, Heavner JE: Incidence and severity of epidural fibrosis after back surgery: an endoscopic study, Pain Pract 2010; 10: 18-24.

[23] Hatten Jr H.: Lumbar epidurography with metrizamide. Radiology 1980; 137:129-136.

[24] Stewart H., Quinnell R., Dann N.: Epidurography in the management of sciatica. $\mathrm{Br} J$ Rheumatol 1987; 26(6):424-429.

[25] Devulder J., Bogaert L., Castille F., et al: Relevance of epidurography and epidural adhesiolysis in chronic failed back surgery patients. Clin J Pain 1995; 11:147-150.

[26] Manchikanti L., Bakhit C., Pampati V.: Role of epidurography in caudal neuroplasty. Pain Digest 1998; 8:277-281.

[27] Day M., Racz G.: Technique of caudal neuroplasty. Pain Digest 1999; 9(4):255-257.

[28] Horlocker T., Wedel D., Benzon H., et al: Regional anesthesia in the anticoagulated patient: defining the risks (the second ASRA Consensus Conference on Neuraxial Anesthesia and Anticoagulation). Reg Anesth Pain Med 2003; 28:172-197.

[29] Omnipaque product insert, Princeton, NJ, Nycomed, Inc.

[30] Isovue product insert, Princeton, NJ, Bracco Diagnostics, Inc.

[31] Hypaque product insert, Princeton, NJ, Amersham Health, Inc.

[32] Conray product insert, Phillipsburg, NJ, Mallinckrodt, Inc.

[33] Racz G., Day M., Heavner J., et al: Hyaluronidase: a review of approved formulations, indications and off-label use in chronic pain management. Expert Opin Biol Ther 2010; 10(1):127-131. 
[34] Teske W., Zirke S., Nottenkamper J., Lichtinger T., Theodoridis T., Kramer J., Schmidt K.: Anatomical and surgical study of volume determination of the anterolateral epidural space nerve root L5/S1 under the aspect of epidural perineural injection in minimal invasive treatment of lumbar nerve root compression. European Spine Journal 2011; 20(4):537-41.

[35] Lauretti G.R., Mattos A.L., Trevellin W., Righeti C.C.F., Resende C.S.: 911 Sacral Neuroplasty for Postlaminectomy Chronic Low Back Pain. European Journal of Pain 2009; 13(S1):S258a-S258.

[36] Matsumoto. Treatment of lower back and leg pain using the Racz Catheter-Matsumoto way via S1 foramen. WIP World Congress, Maastricht; 2014.

[37] Paincast.com. Paincast I Paincast [Internet] 2014.

[38] Racz G.B., Heavner J.E.: Cervical spinal canal loculation and secondary ischemic cord injury-PVCS-perivenous counter spread-danger sign!!. Pain Pract 2008; 8:399-403.

[39] Larkin T., Carragee E., Cohen S.: A novel technique for delivery of epidural steroids and diagnosing the level of nerve root pathology. J Spinal Disord Tech 2003; 16(2): 186-192.

[40] Scanlon G.C., Moeller-Bertram T., Romanowsky S.M., Wallace M.S.: Cervical Transforaminal Epidural Steriod Injections More Dangerous Than We Think?. Spine; 32(11):1249-1256.

[41] Jamison A.E., Hsu E., Cohen S.P.: Epidural adhesiolysis: an evidence based review. J Neurosurg Sci 2014; 58:65-76.

[42] Racz G.B., Heavner J.E., Bosscher H., Helm II S.: The MILD Procedure. Pain Practice 2013; 13(7):594-596.

[43] Racz G.B., Sabonghy M., Gintautas J., et al: Intractable pain therapy using a new type of epidural catheter. JAMA 1985; 248:579-580.

[44] Sakai T., Aoki H., Hojo M., et al: Adhesiolysis and targeted steroid/local anesthetic injection during epiduroscopy alleviates pain and reduces sensory nerve dysfunction in patients with chronic sciatica. J Anesth 2008; 22(3):242-247.

[45] Anderson S., Racz G., Heavener J.: Evolution of epidural lysis of adhesions. Pain Physician 2000; 3(3):262-270.

[46] Racz G., Holubec J.: Lysis of adhesions in the epidural space. In: Raj P., ed. Techniques of neurolysis, Boston: Kluwer Academic; 1989:57-72.

[47] Arthur J., Racz G., Heinrich R., et al: Epidural space: identification of filling defects and lysis of adhesions in the treatment of chronic painful conditions. Abstracts of the 7 th World Congress on Pain, Paris: IASP Publications; 1993. 
[48] Racz G.B., Day M.R., Heavener J.E., Smith J.P.: “The Racz Procedure: Lysis of Epidural Adhesions (Percutaneous Neuroplasty)." Comprehensive Treatment of Chronic Pain by Medical, Interventional, and Integrative Approaches, Ed. Tim Deer. Springer, 2013.

[49] Stolker R., Vervest A., Gerbrand J.: The management of chronic spinal pain by blockades: a revew. Pain 1994; 58:1-19.

[50] Devulder J., Bogaert L., Castille F., et al: Relevance of epidurography and epidural adhesiolysis in chonic failed back surgery patients. Clin J Pain 1995; 11:147-150.

[51] Racz G., Heavner J.: In response to article by Drs. Devulder et al. Clin J Pain 1995; 11:151-154.

[52] Heavner J., Racz G., Raj P.: Percutaneous epidural neuroplasty: prospective evaluation of $0.9 \%$ saline versus $10 \%$ saline with or without hyaluronidase. Reg Anesth Pain Med 1999; 24:202-207.

[53] Manchikanti L., Pakanati R., Bakhit C., et al: Role of adhesiolysis and hypertonic saline neurolysis in management of low back pain: evaluation of modification of the Racz protocol. Pain Digest 1999; 9:91-96.

[54] Manchikanti L., Pampati V., Fellow B., et al: Role of one day epidural adhesiolysis in management of chronic low back pain: a randomized clinical trial. Pain Phys 2001; 4:153-166.

[55] Manchikanti L., Rivera J., Pampati V., et al: One day lumbar adhesiolysis and hypertonic saline neurolysis in treatment of chronic low back pain: a randomized, doubleblinded trial. Pain Phys 2004; 7:177-186.

[56] Manchikanti L., Cash K., McManus C., et al: The preliminary results of a comparative effectiveness of adhesiolysis and caudal epidural injections in managing chronic low back pain secondary to spinal stenosis. Pain Phys 2009; 12(6):E341-E354.

[57] Manchikanti L., Singh V., Cash K., et al: A comparative effectiveness evaluation of percutaneous adhesiolysis and epidural steroid injections in managing lumbar post surgery syndrome. Pain Phys 2009; 12(6):E355-E368.

[58] Veihelmann A., Devens C., Trouiller H., et al: Epidural neuroplasty versus physiotherapy to relieve pain in patients with sciatica: a prospective randomized blinded clinical trial. J Orthop Sci 2006; 11(4):365-369.

[59] Helm II S., Benyamin R., Chopra P., Deer T., Justiz R.: Percutaneous Adhesiolysis in the Management of Chronic Low Back Pain in Post Lumbar Surgery Syndrome and Spinal Stenosis: A Systematic Review. Pain Physician 2012; 15:E435-E62.

[60] Gerdesmeyer L., Lampe R., Veihelmann A., et al: Chronic radiculopathy: use of minimally invasive percutaneous epidural neurolysis according to Racz. Der Schmerz 2005; 19:285-295. 
[61] Gerdesmeyer L., Rechl H., Wagenpfeil S., et al: Minimally invasive epidural neurolysis in chronic radiculopathy: a prospective controlled study to prove effectiveness. Der Orhopade 2003; 32:869-876.

[62] Gerdesmeyer L., Wagenpfeil S., Birkenmaier C., Veihelmann A., Hauschild M., Wagner K., Al Muderis M., Gollwitzer H., Diehl P., Toepfer A.: Percutaneous Epidural Lysis of Adhesions in Chronic Lumbar Radicular Pain: A Randomized, DoubleBlind, Placebo-Controlled Trial. Pain Physician 2013; 16:185-196.

[63] Koh W.U., Choi S.S., Park S.Y., Joo E.Y., Kim S.H., Lee J.D., Shin J.Y., Leem J.G., Shin J.W.: Transforaminal Hypertonic Saline for the Treatment of Lumbar Lateral Canal Stenosis: A Double-Blinded, Randomized, Active-Control Trial. Pain Physician 2013; 16: 197-211.

[64] Manchikanti L., Singh V., Cash K., Pampati V.: Assesment of effectiveness of percutaneous adhesiolysis and caudal epidural injection in managing post lumbar surgery syndrome: 2-year follow-up of a randomized, controlled trial. Journal of Pain Research 2012; 5: 597-608.

[65] Park C.H., Lee S.H.: Effectiveness of Percutaneous Transforaminal Adhesiolysis in Patients with Lumbar Neuroforaminal Spinal Stenosis. Pain Physician 2013; 16: E37E43.

[66] Park E.J., Park S.Y., Lee S.J., Kim N.S., Koh D.Y.: Clinical Outcomes of Epidural Neuroplasty for Cervical Disc Herniation. Journal of Korean Medical Science 2013; 28: 461-465.

[67] Choi E., Nahm F., Lee P.B.: Evaluation of Prognostic Predictors of Percutaneous Adhesiolysis Using a Racz Catheter for Post Lumbar Surgery Syndrome or Spinal Stenosis. Pain Physician 2013; 16:E531-E536.

[68] Manchikanti L., Helm II S., Pampati V., Racz G.B.: Cost Utility Analysis of Percutaneous Adhesiolysis in Managing Pain of Post-Lumbar Surgery Syndrome and Lumbar Central Spinal Stenosis. Pain Practice 2014; doi:10.1111/papr.12195. 
